\title{
Solvability of a general class of two-dimensional hyperbolic-cotangent-type systems of difference equations
}

Stevo Stević, $1,2,3^{*}$

*Correspondence: sstevic@ptt.rs ${ }^{1}$ Mathematical Institute of the Serbian Academy of Sciences, Beograd, Serbia

2Department of Medical Research,

China Medical University Hospital, China Medical University, Taichung, Taiwan, Republic of China Full list of author information is available at the end of the article

\section{第 Springer}

\begin{abstract}
We show that the following class of two-dimensional hyperbolic-cotangent-type systems of difference equations

$$
x_{n+1}=\frac{u_{n-k} v_{n-1}+a}{u_{n-k}+v_{n-1}}, \quad y_{n+1}=\frac{w_{n-k} s_{n-1}+a}{w_{n-k}+s_{n-1}}, \quad n \in \mathbb{N}_{0}
$$

where $k, l \in \mathbb{N}_{0}, a \in \mathbb{C}, u_{-j}, w_{-j} \in \mathbb{C}, j=\overline{1, k}, v_{-j^{\prime}}, s_{-j^{\prime}}, j^{\prime}=\overline{1, l}$, and each of the sequences $u_{n}, v_{n}, w_{n}, s_{n}$ is equal to $x_{n}$ or $y_{n}$, is theoretically solvable. When $k=0$ and $l=1$, we show that the systems are practically solvable by presenting closed-form formulas for their solutions. To do this, we employ a constructive method, which is possible to use on the complex domain, presenting in this way a new and elegant solution to the problem in this case, and giving a hint how such type of systems can be solved.
\end{abstract}

MSC: 39A45

Keywords: System of difference equations; Product-type system; General solution; Closed-form formula

\section{Introduction}

Let $\mathbb{N}=\{1,2,3, \ldots\}, \mathbb{N}_{0}=\mathbb{N} \cup\{0\}, \mathbb{Z}$ be the set of whole numbers, $\mathbb{R}$ be the set of reals, and $\mathbb{C}$ of complex numbers. If we write $j=\overline{n_{1}, n_{2}}$, where $n_{1}, n_{2} \in \mathbb{Z}$, it means that $j$ takes the whole numbers such that $n_{1} \leq j \leq n_{2}$.

\subsection{A little history and some useful classical results}

Motivated by some concrete problems from combinatorics, probability, economics, and other branches of mathematics and science, researchers of the eighteenth century started investigating recurrence relations (the notion coined in [1]), known also as difference equations. Natural connection between recurrence relations and difference equations has been noticed long time ago, for example, in [2]. One of the basic natural problems is to find some (nonrecursive) expressions for solutions to difference equations and systems of difference equations in as much as simpler forms (the problem is to try to find formulas for solutions to the equations and systems in closed form).

(c) The Author(s) 2019. This article is distributed under the terms of the Creative Commons Attribution 4.0 International License (http://creativecommons.org/licenses/by/4.0/), which permits unrestricted use, distribution, and reproduction in any medium, provided you give appropriate credit to the original author(s) and the source, provide a link to the Creative Commons license, and indicate if changes were made. 
Closed-form formulas for solutions to homogeneous linear difference equations with constant coefficients, that is, for the equations of the form

$$
x_{n}=a_{1} x_{n-1}+a_{2} x_{n-2}+\cdots+a_{k} x_{n-k}, \quad n \in \mathbb{N}_{0},
$$

where $a_{j} \in \mathbb{R}$ (or $\mathbb{C}$ ), $j=\overline{1, k}, a_{k} \neq 0$, for small values of $k$, can be found in old books [3, 4], whereas a pretty complete theory on the equations was established some time later (see, e.g., [5-7]).

It was shown that the general solution to difference equation (1) has the following form:

$$
x_{n}=\sum_{j=1}^{m} Q_{j}(n) \lambda_{j}^{n},
$$

where $\lambda_{j}, j=\overline{1, m}$, are the roots of the polynomial

$$
P_{k}(\lambda)=\lambda^{k}-a_{1} \lambda^{k-1}-a_{2} \lambda^{k-2}-\cdots-a_{k-1} \lambda-a_{k},
$$

associated with equation (1) (characteristic polynomial), whereas $Q_{j}(n), j=\overline{1, m}$, are polynomials of degrees $d_{j}=r_{j}-1, j=\overline{1, m}$, respectively, where $r_{j}$ is the multiplicity of the root $\lambda_{j}$.

Bearing in mind the literature from the mid of the previous century (see, for example, [8-12]), it seems there had not been much novelties in solvability theory of difference equations for a long time.

\subsection{On recent topics of interest and general motivations}

Recent progress in computer science has had some impact on the research area. It has increased interest in recurrent relations; on the other hand, it has caused also some, a bit problematic, non-theoretical ways for investigating the relations. Namely, many recent papers on solvability of difference equations and systems consider some equations and systems which are close cousins to well-known solvable ones. Some comments related to it, as well as theoretical explanations, can be found, for example, in [13-18]. We have noticed the problem yet in 2004 on the example of the equation

$$
x_{n+2}=\frac{x_{n}}{c x_{n+1} x_{n}+d}, \quad n \in \mathbb{N}_{0} .
$$

Nevertheless, it motivated some further investigations on extensions of equation (4) (see, e.g., [19-24]; see also [25] which treats equation (4) in another way).

The systems of difference equations studied in some of these papers are usually symmetric, that is, of the following form:

$$
\begin{aligned}
& u_{n}=f\left(u_{n-s_{1}}, \ldots, u_{n-s_{k}}, v_{n-t_{1}}, \ldots, v_{n-t_{l}}\right), \\
& v_{n}=f\left(v_{n-s_{1}}, \ldots, v_{n-s_{k}}, u_{n-t_{1}}, \ldots, u_{n-t_{l}}\right),
\end{aligned}
$$

for $n \in \mathbb{N}_{0}$, or close to symmetric ones, which is an area that attracted some attention since the end of the previous century (see, e.g., [26-42]). In some of these papers invariants, a 
topic close to solvability, were studied (see papers [27-29, 31, 33, 34] by Papaschinopoulos and Schinas). For a recent, quite unexpected, use of invariants in solvability of a class of difference equations, see [43].

As we have already mentioned, difference equations, as well as systems of difference equations, appear in many practical problems as discrete models. Many of them are solvable (see, for example, some of them in $[2,5,8,9,11,44-55]$ ).

\subsection{Previous investigations leading to the one in this paper}

One of the difference equations whose special cases attracted some attention in the last few decades is the following:

$$
x_{n+1}=\frac{x_{n-k} x_{n-l}+a}{x_{n-k}+x_{n-l}}, \quad n \in \mathbb{N}_{0},
$$

where $k, l \in \mathbb{N}_{0}, a \in \mathbb{R}$.

Employing a result from [56], in [57] we proved a global stability result for positive solutions to a generalization of equation (5), showing that a previously obtained result in the case $k=0, l=1$ is essentially known. Motivated by the same result from [56], Papaschinopoulos and Schinas in [58] presented a related global stability result for systems of difference equations, which was improved in [59]. Equation (5) with $a \neq 0$ is easily reduced to the case $a=1$, which looks like a familiar formula for trigonometric functions. That difference equations obtained by using known trigonometric relations could be solvable has been known for a long time (see, e.g., [6]). It has been our impression, for some time, that the solvability of equation (5) in the case $k=0, l=1$, had been known for a long time and that it could be found easily in the literature. The fact that we could not find an expected natural solution to the problem in the literature, and that we have only found a recent solution to the problem of different type [60], motivated us to present the natural solution recently in [61].

Following one of the usual ways for generalizing results on scalar difference equations to close-to-symmetric systems of difference equations, quite recently in [62] and [63] we have considered solvability problem for the following systems of difference equations:

$$
x_{n+1}=\frac{u_{n} v_{n-1}+a}{u_{n}+v_{n-1}}, \quad y_{n+1}=\frac{w_{n} s_{n-1}+a}{w_{n}+s_{n-1}}, \quad n \in \mathbb{N}_{0},
$$

where $a$ and $u_{j}, v_{j}, w_{j}, s_{j}, j=-1,0$, are complex numbers, whereas $u_{n}, v_{n}, w_{n}, s_{n}$ are equal to $x_{n}$ or $y_{n}$. In fact, our original aim in [62] and [63] was essentially to study real solutions to some of the systems in (6), which is the main reason why some methods for dealing with real functions have been predominately used therein, while the solvability results for the case of complex parameters and initial values have been obtained indirectly.

The motivation for the study of the systems in (6) stemmed from the systems in our previous papers [20] and [64], where the solvability of the following systems:

$$
x_{n+1}=\frac{u_{n}}{1+v_{n}}, \quad y_{n+1}=\frac{w_{n}}{1+s_{n}}, \quad n \in \mathbb{N}_{0},
$$

where $u_{n}, v_{n}, w_{n}, s_{n}$ are some of the sequences $x_{n}$ or $y_{n}$, was studied. We have shown an interesting result therein, namely that fourteen systems out of these sixteen ones are solvable and presented closed-form formulas for their solutions. It is also interesting to 
mention that our motivation for the study of the systems in (7) stemmed from our previous investigations on the long-term behavior of solutions to the general difference equation

$$
x_{n}=f\left(x_{n-1}, x_{n-2}, \ldots, x_{n-k}\right), \quad n \in \mathbb{N}_{0},
$$

where the function $f$ satisfies the following condition: $f(t, t, \ldots, t) \sim t$, as $t \rightarrow 0$. When $f(t)=\frac{t}{1+b t}$, we have $f(t) \sim t$, as $t \rightarrow 0$, and a simple solvable difference equation is obtained (see, e.g, [6, 65]), which suggested us that some of the systems in (7) could be also solvable.

Bearing in mind that there is a method for solving equation (5) which directly deals with the case of complex parameters and initial values [60], it is a natural problem to try to solve some of the corresponding close-to-symmetric systems of difference equations in a related way.

\subsection{The Fibonacci sequence}

Here we say a few words on the sequence that will be frequently used in the rest of the paper.

The sequence satisfying the following recurrence relation:

$$
a_{n+2}=a_{n+1}+a_{n}, \quad n \in \mathbb{N}
$$

and such that $a_{1}=a_{2}=1$, is called the Fibonacci sequence (see, e.g., $\left.[45,48,66,67]\right)$. Here, as usual, we denote the sequence as $f_{n}$.

From the de Moivre formula for solutions to linear homogeneous difference equations with constant coefficients $[3,4]$, we have

$$
f_{n}=\frac{\lambda_{1}^{n}-\lambda_{2}^{n}}{\lambda_{1}-\lambda_{2}}=\frac{\left(\frac{1+\sqrt{5}}{2}\right)^{n}-\left(\frac{1-\sqrt{5}}{2}\right)^{n}}{\sqrt{5}}, \quad n \in \mathbb{N}
$$

where

$$
\lambda_{1,2}=\frac{1 \pm \sqrt{5}}{2} .
$$

It should be easily checked that formula (9) holds for every $n \in \mathbb{Z}$ (see, e.g., [66]).

\subsection{Aim of this paper, types of solvabilities, and main methods}

One of our aims here is to show that the following class of nonlinear two-dimensional systems of difference equations:

$$
x_{n+1}=\frac{u_{n-k} v_{n-l}+a}{u_{n-k}+v_{n-l}}, \quad y_{n+1}=\frac{w_{n-k} s_{n-l}+a}{w_{n-k}+s_{n-l}}, \quad n \in \mathbb{N}_{0},
$$

where $k, l \in \mathbb{N}_{0}, a, u_{-j}, w_{-j}, j=\overline{1, k}, v_{-j^{\prime}}, s_{-j^{\prime}}, j^{\prime}=\overline{1, l}$, are complex numbers, and each of the sequences $u_{n}, v_{n}, w_{n}, s_{n}$ is equal to $x_{n}$ or $y_{n}$, is theoretically solvable.

As we have already mentioned, to each difference equation of the form in (1) is associated the polynomial (3). It is known that if the order of a polynomial is greater or equal to five, it need not be solvable by radicals [68]. Since general solution to equation (1) is given by formula (2), in this case we might not be able to find all the roots of polynomial 
(3) by radicals. Hence, in such cases we say that equation (1) is theoretically solvable. If the order is strictly less than five, then by using known formulas/methods for solving polynomials by radicals we can find all the roots, from which along with formula (2) we have an explicit closed-form formula for solutions to equation (1), and we say that the equation is practically solvable in this case. Hence, for each difference equation or system which can be solved by using equation (1) with order greater or equal to five, we say that it is theoretically solvable, whereas if the order of the polynomial is strictly less than five, we say that such an equation or system is practically solvable. Note that if a polynomial of order strictly bigger than four is of a specific type, then it can be solvable by radicals, so in such special cases a specific equation or system can be practically solvable. Hence, for theoretical solvability it is important that the polynomial (3) has general form.

Since a system of difference equations can have initial values for which solutions are not defined, but they usually do not affect the solvability, we regard that a system of difference equations is solvable in closed form if there is a finite number of closed-form formulas from which any well-defined solution to the system can be obtained.

As a concrete example, we study here system (11) in the case $k=0$ and $l=1$ in detail, and show that it is practically solvable, presenting a new constructive and straightforward solution to the solvability problem unlike the solutions given in [62] and [63].

To do this, the methods from papers on product-type difference equations and systems of difference equations will be considerably used (see, e.g., $[35-42,69]$ and the references therein).

\section{Case $a=0$}

This section is devoted to studying solvability of system (11) in the case $a=0$. In this case system (11) becomes

$$
x_{n+1}=\frac{u_{n-k} v_{n-l}}{u_{n-k}+v_{n-l}}, \quad y_{n+1}=\frac{w_{n-k} s_{n-l}}{w_{n-k}+s_{n-l}}, \quad n \in \mathbb{N}_{0},
$$

where $k, l \in \mathbb{N}_{0}$.

By using the changes of variables

$$
\hat{x}_{n}=\frac{1}{x_{n}}, \quad \hat{y}_{n}=\frac{1}{y_{n}}, \quad \hat{u}_{n}=\frac{1}{u_{n}}, \quad \hat{v}_{n}=\frac{1}{v_{n}}, \quad \hat{w}_{n}=\frac{1}{w_{n}}, \quad \hat{s}_{n}=\frac{1}{s_{n}},
$$

system (12) can be written in the following form:

$$
\hat{x}_{n+1}=\hat{u}_{n-k}+\hat{v}_{n-l}, \quad \hat{y}_{n+1}=\hat{w}_{n-k}+\hat{s}_{n-l}, \quad n \in \mathbb{N}_{0} .
$$

Depending on which values sequences $u_{n}, v_{n}, w_{n}, s_{n}$ have $\left(x_{n}\right.$ or $\left.y_{n}\right)$, there are sixteen possible systems. They are the following:

$$
\begin{array}{ll}
\hat{x}_{n+1}=\hat{x}_{n-k}+\hat{x}_{n-l}, & \hat{y}_{n+1}=\hat{x}_{n-k}+\hat{x}_{n-l} ; \\
\hat{x}_{n+1}=\hat{x}_{n-k}+\hat{x}_{n-l}, & \hat{y}_{n+1}=\hat{y}_{n-k}+\hat{x}_{n-l} ; \\
\hat{x}_{n+1}=\hat{x}_{n-k}+\hat{x}_{n-l}, & \hat{y}_{n+1}=\hat{x}_{n-k}+\hat{y}_{n-l} ; \\
\hat{x}_{n+1}=\hat{x}_{n-k}+\hat{x}_{n-l}, & \hat{y}_{n+1}=\hat{y}_{n-k}+\hat{y}_{n-l} ;
\end{array}
$$




$$
\begin{array}{ll}
\hat{x}_{n+1}=\hat{x}_{n-k}+\hat{y}_{n-l}, & \hat{y}_{n+1}=\hat{x}_{n-k}+\hat{x}_{n-l} ; \\
\hat{x}_{n+1}=\hat{x}_{n-k}+\hat{y}_{n-l}, & \hat{y}_{n+1}=\hat{y}_{n-k}+\hat{x}_{n-l} ; \\
\hat{x}_{n+1}=\hat{x}_{n-k}+\hat{y}_{n-l}, & \hat{y}_{n+1}=\hat{x}_{n-k}+\hat{y}_{n-l} ; \\
\hat{x}_{n+1}=\hat{x}_{n-k}+\hat{y}_{n-l}, & \hat{y}_{n+1}=\hat{y}_{n-k}+\hat{y}_{n-l} ; \\
\hat{x}_{n+1}=\hat{y}_{n-k}+\hat{x}_{n-l}, & \hat{y}_{n+1}=\hat{x}_{n-k}+\hat{x}_{n-l} ; \\
\hat{x}_{n+1}=\hat{y}_{n-k}+\hat{x}_{n-l}, & \hat{y}_{n+1}=\hat{y}_{n-k}+\hat{x}_{n-l} ; \\
\hat{x}_{n+1}=\hat{y}_{n-k}+\hat{x}_{n-l}, & \hat{y}_{n+1}=\hat{x}_{n-k}+\hat{y}_{n-l} ; \\
\hat{x}_{n+1}=\hat{y}_{n-k}+\hat{x}_{n-l}, & \hat{y}_{n+1}=\hat{y}_{n-k}+\hat{y}_{n-l} ; \\
\hat{x}_{n+1}=\hat{y}_{n-k}+\hat{y}_{n-l}, & \hat{y}_{n+1}=\hat{x}_{n-k}+\hat{x}_{n-l} ; \\
\hat{x}_{n+1}=\hat{y}_{n-k}+\hat{y}_{n-l}, & \hat{y}_{n+1}=\hat{y}_{n-k}+\hat{x}_{n-l} ; \\
\hat{x}_{n+1}=\hat{y}_{n-k}+\hat{y}_{n-l}, & \hat{y}_{n+1}=\hat{x}_{n-k}+\hat{y}_{n-l} ; \\
\hat{x}_{n+1}=\hat{y}_{n-k}+\hat{y}_{n-l}, & \hat{y}_{n+1}=\hat{y}_{n-k}+\hat{y}_{n-l}
\end{array}
$$

for $n \in \mathbb{N}_{0}$.

Our first theorem deals with the theoretical solvability of system (11) in the case $a=0$, that is, of system (12).

Theorem 1 Assume that $a=0$. Then system (11) is (theoretically) solvable.

Proof To do this, we need to prove the theoretical solvability of systems (15)-(30).

Case 1, system (15). The first equation in system (15) is linear with constant coefficients, so it is theoretically solvable. Since from (15) it immediately follows that $y_{n}=x_{n}, n \in \mathbb{N}$, the solvability of the system follows.

Case 2, system (16). Recall that the first equation in system (16) is solvable. Moreover, we know that its general solution has the form in (2), where $\lambda_{j}, j=\overline{1, m}$, are the characteristic roots of the characteristic polynomial associated with the equation, while $Q_{j}, j=\overline{1, m}$, are some polynomials of degrees $d_{j}=r_{j}-1, j=\overline{1, m}$, respectively, where $r_{j}$ is the multiplicity of the characteristic root $\lambda_{j}$.

From this and the second equation we have

$$
\hat{y}_{n+1}-\hat{y}_{n-k}=\sum_{j=1}^{m} Q_{j}(n-l) \lambda_{1}^{n-l},
$$

which is a nonhomogeneous linear difference equation with constant coefficients whose nonhomogeneous part has the polynomial-exponential form. It is well known that such difference equations are solvable (see, e.g., [8-11]). Hence, system (16) is solvable.

Case 3, system (17). This case is essentially the same as Case 2, with the only difference that the letters $k$ and $l$ are interchanged in the second equation. Hence, the system is also solvable.

Case 4, system (18). This system consists of two copies of the same linear difference equation with constant coefficients, so it is obviously solvable. 
Case 5, system (19). Using the second equation in (19) into the first one, we get

$$
\hat{x}_{n+1}-\hat{x}_{n-k}-\hat{x}_{n-k-l-1}-\hat{x}_{n-2 l-1}=0,
$$

which is a linear difference equation with constant coefficients, hence solvable. Using the general solution to equation (32) in the second equation in (19) it is obtained a closed-form formula for $y_{n}$. Hence, system (19) is solvable.

Case 6, system (20). From the first equation in (20) we have

$$
\hat{y}_{n}=\hat{x}_{n+l+1}-\hat{x}_{n+l-k} \text {. }
$$

Using (33) into the second equation in (20) we obtain

$$
\hat{x}_{n+l+2}-2 \hat{x}_{n+l-k+1}+\hat{x}_{n+l-2 k}-\hat{x}_{n-l}=0
$$

which is a linear difference equation with constant coefficients. Hence, its general solution can be found. Using the general solution to equation (34) in (33), a closed-form formula for $y_{n}$ is obtained, from which the solvability of system (20) follows.

Case 7, system (21). This case is essentially the same as Case 6, with the only difference that the letters $k$ and $l$ are interchanged in the second equation. Hence, the system is also solvable.

Case 8, system (22). This system is obtained from (16) when the letters $x$ and $y$ are interchanged, from which its solvability follows.

Case 9, system (23). This case is essentially the same as Case 5 , with the only difference that the letters $k$ and $l$ are interchanged in the first equation. Hence, the system is also solvable.

Case 10, system (24). This case is essentially the same as Case 6, with the only difference that the letters $k$ and $l$ are interchanged in the first equation. Hence, the system is also solvable.

Case 11, system (25). This case is essentially the same as Case 7, with the only difference that the letters $k$ and $l$ are interchanged in the first equation. Hence, the system is also solvable.

Case 12, system (26). This case is essentially the same as Case 8 , with the only difference that the letters $k$ and $l$ are interchanged in the first equation. Hence, the system is also solvable.

Case 13, system (27). Using the first equation in (27) into the second one, we obtain

$$
\hat{y}_{n+1}-\hat{y}_{n-2 k-1}-2 \hat{y}_{n-k-l-1}-\hat{y}_{n-2 l-1},
$$

which is a linear difference equation with constant coefficients. Hence, its general solution can be found. Using the general solution to equation (35) in the first equation in (27), a closed-form formula for $x_{n}$ is obtained, from which the solvability of system (27) follows.

Case 14, system (28). This system is obtained from (19) when the letters $x$ and $y$ are interchanged, from which its solvability follows.

Case 15, system (29). This system is obtained from system (23) when the letters $x$ and $y$ are interchanged, from which its solvability follows. 
Case 16, system (30). This system is obtained from system (15) when the letters $x$ and $y$ are interchanged, from which its solvability follows.

Remark 1 It should be pointed out that all the linear transformations used in the proof of Theorem 1 transform original systems of difference equations to equivalent ones, from which it follows that there is no possibility of getting new or loosing some existing solutions. The changes of variables (13) do not transform the systems in (12) to equivalent ones. Nevertheless, since, by the definition, we take into consideration only well-defined solutions to the systems, by using the changes all such solutions are obtained.

\section{Case $a \neq 0$}

In this section we consider system of difference equations (11) in the case $a \in \mathbb{C} \backslash\{0\}$.

First, note that from (11) it follows that

$$
\begin{aligned}
& x_{n+1}+\sqrt{a}=\frac{\left(u_{n-k}+\sqrt{a}\right)\left(v_{n-l}+\sqrt{a}\right)}{u_{n-k}+v_{n-l}}, \\
& y_{n+1}+\sqrt{a}=\frac{\left(w_{n-k}+\sqrt{a}\right)\left(s_{n-l}+\sqrt{a}\right)}{w_{n-k}+s_{n-l}}
\end{aligned}
$$

for $n \in \mathbb{N}_{0}$, and

$$
\begin{aligned}
& x_{n+1}-\sqrt{a}=\frac{\left(u_{n-k}-\sqrt{a}\right)\left(v_{n-l}-\sqrt{a}\right)}{u_{n-k}+v_{n-l}}, \\
& y_{n+1}-\sqrt{a}=\frac{\left(w_{n-k}-\sqrt{a}\right)\left(s_{n-l}-\sqrt{a}\right)}{w_{n-k}+s_{n-l}}
\end{aligned}
$$

for every $n \in \mathbb{N}_{0}$, where $\sqrt{a}$ is one of the two roots of the polynomial equation $z^{2}+a=0$.

From (36) and (37) it follows that

$$
\begin{aligned}
& \frac{x_{n+1}+\sqrt{a}}{x_{n+1}-\sqrt{a}}=\frac{u_{n-k}+\sqrt{a}}{u_{n-k}-\sqrt{a}} \cdot \frac{v_{n-l}+\sqrt{a}}{v_{n-l}-\sqrt{a}} \\
& \frac{y_{n+1}+\sqrt{a}}{y_{n+1}-\sqrt{a}}=\frac{w_{n-k}+\sqrt{a}}{w_{n-k}-\sqrt{a}} \cdot \frac{s_{n-l}+\sqrt{a}}{s_{n-l}-\sqrt{a}}
\end{aligned}
$$

for $n \in \mathbb{N}_{0}$.

Depending on which values sequences $u_{n}, v_{n}, w_{n}, s_{n}$ have $\left(x_{n}\right.$ or $\left.y_{n}\right)$, there are the following sixteen cases:

$$
\begin{aligned}
& \frac{x_{n+1}+\sqrt{a}}{x_{n+1}-\sqrt{a}}=\frac{x_{n-k}+\sqrt{a}}{x_{n-k}-\sqrt{a}} \cdot \frac{x_{n-l}+\sqrt{a}}{x_{n-l}-\sqrt{a}}, \\
& \frac{y_{n+1}+\sqrt{a}}{y_{n+1}-\sqrt{a}}=\frac{x_{n-k}+\sqrt{a}}{x_{n-k}-\sqrt{a}} \cdot \frac{x_{n-l}+\sqrt{a}}{x_{n-l}-\sqrt{a}} ; \\
& \frac{x_{n+1}+\sqrt{a}}{x_{n+1}-\sqrt{a}}=\frac{x_{n-k}+\sqrt{a}}{x_{n-k}-\sqrt{a}} \cdot \frac{x_{n-l}+\sqrt{a}}{x_{n-l}-\sqrt{a}}, \\
& \frac{y_{n+1}+\sqrt{a}}{y_{n+1}-\sqrt{a}}=\frac{y_{n-k}+\sqrt{a}}{y_{n-k}-\sqrt{a}} \cdot \frac{x_{n-l}+\sqrt{a}}{x_{n-l}-\sqrt{a}} ;
\end{aligned}
$$




$$
\begin{aligned}
& \frac{x_{n+1}+\sqrt{a}}{x_{n+1}-\sqrt{a}}=\frac{x_{n-k}+\sqrt{a}}{x_{n-k}-\sqrt{a}} \cdot \frac{x_{n-l}+\sqrt{a}}{x_{n-l}-\sqrt{a}}, \\
& \frac{y_{n+1}+\sqrt{a}}{y_{n+1}-\sqrt{a}}=\frac{x_{n-k}+\sqrt{a}}{x_{n-k}-\sqrt{a}} \cdot \frac{y_{n-l}+\sqrt{a}}{y_{n-l}-\sqrt{a}} \text {; } \\
& \frac{x_{n+1}+\sqrt{a}}{x_{n+1}-\sqrt{a}}=\frac{x_{n-k}+\sqrt{a}}{x_{n-k}-\sqrt{a}} \cdot \frac{x_{n-l}+\sqrt{a}}{x_{n-l}-\sqrt{a}}, \\
& \frac{y_{n+1}+\sqrt{a}}{y_{n+1}-\sqrt{a}}=\frac{y_{n-k}+\sqrt{a}}{y_{n-k}-\sqrt{a}} \cdot \frac{y_{n-l}+\sqrt{a}}{y_{n-l}-\sqrt{a}} \text {; } \\
& \frac{x_{n+1}+\sqrt{a}}{x_{n+1}-\sqrt{a}}=\frac{y_{n-k}+\sqrt{a}}{y_{n-k}-\sqrt{a}} \cdot \frac{x_{n-l}+\sqrt{a}}{x_{n-l}-\sqrt{a}}, \\
& \frac{y_{n+1}+\sqrt{a}}{y_{n+1}-\sqrt{a}}=\frac{x_{n-k}+\sqrt{a}}{x_{n-k}-\sqrt{a}} \cdot \frac{x_{n-l}+\sqrt{a}}{x_{n-l}-\sqrt{a}} \text {; } \\
& \frac{x_{n+1}+\sqrt{a}}{x_{n+1}-\sqrt{a}}=\frac{y_{n-k}+\sqrt{a}}{y_{n-k}-\sqrt{a}} \cdot \frac{x_{n-l}+\sqrt{a}}{x_{n-l}-\sqrt{a}}, \\
& \frac{y_{n+1}+\sqrt{a}}{y_{n+1}-\sqrt{a}}=\frac{y_{n-k}+\sqrt{a}}{y_{n-k}-\sqrt{a}} \cdot \frac{x_{n-l}+\sqrt{a}}{x_{n-l}-\sqrt{a}} ; \\
& \frac{x_{n+1}+\sqrt{a}}{x_{n+1}-\sqrt{a}}=\frac{y_{n-k}+\sqrt{a}}{y_{n-k}-\sqrt{a}} \cdot \frac{x_{n-l}+\sqrt{a}}{x_{n-l}-\sqrt{a}} \text {, } \\
& \frac{y_{n+1}+\sqrt{a}}{y_{n+1}-\sqrt{a}}=\frac{x_{n-k}+\sqrt{a}}{x_{n-k}-\sqrt{a}} \cdot \frac{y_{n-l}+\sqrt{a}}{y_{n-l}-\sqrt{a}} \text {; } \\
& \frac{x_{n+1}+\sqrt{a}}{x_{n+1}-\sqrt{a}}=\frac{y_{n-k}+\sqrt{a}}{y_{n-k}-\sqrt{a}} \cdot \frac{x_{n-l}+\sqrt{a}}{x_{n-l}-\sqrt{a}}, \\
& \frac{y_{n+1}+\sqrt{a}}{y_{n+1}-\sqrt{a}}=\frac{y_{n-k}+\sqrt{a}}{y_{n-k}-\sqrt{a}} \cdot \frac{y_{n-l}+\sqrt{a}}{y_{n-l}-\sqrt{a}} \text {; } \\
& \frac{x_{n+1}+\sqrt{a}}{x_{n+1}-\sqrt{a}}=\frac{x_{n-k}+\sqrt{a}}{x_{n-k}-\sqrt{a}} \cdot \frac{y_{n-l}+\sqrt{a}}{y_{n-l}-\sqrt{a}}, \\
& \frac{y_{n+1}+\sqrt{a}}{y_{n+1}-\sqrt{a}}=\frac{x_{n-k}+\sqrt{a}}{x_{n-k}-\sqrt{a}} \cdot \frac{x_{n-l}+\sqrt{a}}{x_{n-l}-\sqrt{a}} ; \\
& \frac{x_{n+1}+\sqrt{a}}{x_{n+1}-\sqrt{a}}=\frac{x_{n-k}+\sqrt{a}}{x_{n-k}-\sqrt{a}} \cdot \frac{y_{n-l}+\sqrt{a}}{y_{n-l}-\sqrt{a}}, \\
& \frac{y_{n+1}+\sqrt{a}}{y_{n+1}-\sqrt{a}}=\frac{y_{n-k}+\sqrt{a}}{y_{n-k}-\sqrt{a}} \cdot \frac{x_{n-l}+\sqrt{a}}{x_{n-l}-\sqrt{a}} \text {; } \\
& \frac{x_{n+1}+\sqrt{a}}{x_{n+1}-\sqrt{a}}=\frac{x_{n-k}+\sqrt{a}}{x_{n-k}-\sqrt{a}} \cdot \frac{y_{n-l}+\sqrt{a}}{y_{n-l}-\sqrt{a}}, \\
& \frac{y_{n+1}+\sqrt{a}}{y_{n+1}-\sqrt{a}}=\frac{x_{n-k}+\sqrt{a}}{x_{n-k}-\sqrt{a}} \cdot \frac{y_{n-l}+\sqrt{a}}{y_{n-l}-\sqrt{a}} ; \\
& \frac{x_{n+1}+\sqrt{a}}{x_{n+1}-\sqrt{a}}=\frac{x_{n-k}+\sqrt{a}}{x_{n-k}-\sqrt{a}} \cdot \frac{y_{n-l}+\sqrt{a}}{y_{n-l}-\sqrt{a}}, \\
& \frac{y_{n+1}+\sqrt{a}}{y_{n+1}-\sqrt{a}}=\frac{y_{n-k}+\sqrt{a}}{y_{n-k}-\sqrt{a}} \cdot \frac{y_{n-l}+\sqrt{a}}{y_{n-l}-\sqrt{a}} \text {; } \\
& \frac{x_{n+1}+\sqrt{a}}{x_{n+1}-\sqrt{a}}=\frac{y_{n-k}+\sqrt{a}}{y_{n-k}-\sqrt{a}} \cdot \frac{y_{n-l}+\sqrt{a}}{y_{n-l}-\sqrt{a}}, \\
& \frac{y_{n+1}+\sqrt{a}}{y_{n+1}-\sqrt{a}}=\frac{x_{n-k}+\sqrt{a}}{x_{n-k}-\sqrt{a}} \cdot \frac{x_{n-l}+\sqrt{a}}{x_{n-l}-\sqrt{a}} ;
\end{aligned}
$$




$$
\begin{aligned}
& \frac{x_{n+1}+\sqrt{a}}{x_{n+1}-\sqrt{a}}=\frac{y_{n-k}+\sqrt{a}}{y_{n-k}-\sqrt{a}} \cdot \frac{y_{n-l}+\sqrt{a}}{y_{n-l}-\sqrt{a}}, \\
& \frac{y_{n+1}+\sqrt{a}}{y_{n+1}-\sqrt{a}}=\frac{y_{n-k}+\sqrt{a}}{y_{n-k}-\sqrt{a}} \cdot \frac{x_{n-l}+\sqrt{a}}{x_{n-l}-\sqrt{a}} ; \\
& \frac{x_{n+1}+\sqrt{a}}{x_{n+1}-\sqrt{a}}=\frac{y_{n-k}+\sqrt{a}}{y_{n-k}-\sqrt{a}} \cdot \frac{y_{n-l}+\sqrt{a}}{y_{n-l}-\sqrt{a}} \\
& \frac{y_{n+1}+\sqrt{a}}{y_{n+1}-\sqrt{a}}=\frac{x_{n-k}+\sqrt{a}}{x_{n-k}-\sqrt{a}} \cdot \frac{y_{n-l}+\sqrt{a}}{y_{n-l}-\sqrt{a}} ; \\
& \frac{x_{n+1}+\sqrt{a}}{x_{n+1}-\sqrt{a}}=\frac{y_{n-k}+\sqrt{a}}{y_{n-k}-\sqrt{a}} \cdot \frac{y_{n-l}+\sqrt{a}}{y_{n-l}-\sqrt{a}} \\
& \frac{y_{n+1}+\sqrt{a}}{y_{n+1}-\sqrt{a}}=\frac{y_{n-k}+\sqrt{a}}{y_{n-k}-\sqrt{a}} \cdot \frac{y_{n-l}+\sqrt{a}}{y_{n-l}-\sqrt{a}}
\end{aligned}
$$

for $n \in \mathbb{N}_{0}$.

Using the changes of variables

$$
\zeta_{n}=\frac{x_{n}+\sqrt{a}}{x_{n}-\sqrt{a}}, \quad \eta_{n}=\frac{y_{n}+\sqrt{a}}{y_{n}-\sqrt{a}},
$$

systems (40)-(55) become

$$
\begin{aligned}
& \zeta_{n+1}=\zeta_{n-k} \zeta_{n-l}, \quad \eta_{n+1}=\zeta_{n-k} \zeta_{n-l}, \\
& \zeta_{n+1}=\zeta_{n-k} \zeta_{n-l}, \quad \eta_{n+1}=\eta_{n-k} \zeta_{n-l}, \\
& \zeta_{n+1}=\zeta_{n-k} \zeta_{n-l}, \quad \eta_{n+1}=\zeta_{n-k} \eta_{n-l}, \\
& \zeta_{n+1}=\zeta_{n-k} \zeta_{n-l}, \quad \eta_{n+1}=\eta_{n-k} \eta_{n-l}, \\
& \zeta_{n+1}=\eta_{n-k} \zeta_{n-l}, \quad \eta_{n+1}=\zeta_{n-k} \zeta_{n-l}, \\
& \zeta_{n+1}=\eta_{n-k} \zeta_{n-l}, \quad \eta_{n+1}=\eta_{n-k} \zeta_{n-l}, \\
& \zeta_{n+1}=\eta_{n-k} \zeta_{n-l}, \quad \eta_{n+1}=\zeta_{n-k} \eta_{n-l}, \\
& \zeta_{n+1}=\eta_{n-k} \zeta_{n-l}, \quad \eta_{n+1}=\eta_{n-k} \eta_{n-l}, \\
& \zeta_{n+1}=\zeta_{n-k} \eta_{n-l}, \quad \eta_{n+1}=\zeta_{n-k} \zeta_{n-l}, \\
& \zeta_{n+1}=\zeta_{n-k} \eta_{n-l}, \quad \eta_{n+1}=\eta_{n-k} \zeta_{n-l}, \\
& \zeta_{n+1}=\zeta_{n-k} \eta_{n-l}, \quad \eta_{n+1}=\zeta_{n-k} \eta_{n-l}, \\
& \zeta_{n+1}=\zeta_{n-k} \eta_{n-l}, \quad \eta_{n+1}=\eta_{n-k} \eta_{n-l}, \\
& \zeta_{n+1}=\eta_{n-k} \eta_{n-l}, \quad \eta_{n+1}=\zeta_{n-k} \zeta_{n-l}, \\
& \zeta_{n+1}=\eta_{n-k} \eta_{n-l}, \quad \eta_{n+1}=\eta_{n-k} \zeta_{n-l}, \\
& \zeta_{n+1}=\eta_{n-k} \eta_{n-l}, \quad \eta_{n+1}=\zeta_{n-k} \eta_{n-l}, \\
& \zeta_{n+1}=\eta_{n-k} \eta_{n-l}, \quad \eta_{n+1}=\eta_{n-k} \eta_{n-l},
\end{aligned}
$$

for $n \in \mathbb{N}_{0}$, respectively. 
Systems (57)-(72) are product-type ones. A systematic study of practical solvability of product-type systems of difference equations has been conducted, for example, in our recent papers [35-41] (see also the related references therein).

Note that from (56) we have

$$
x_{n}=\sqrt{a} \frac{\zeta_{n}+1}{\zeta_{n}-1}, \quad y_{n}=\sqrt{a} \frac{\eta_{n}+1}{\eta_{n}-1} .
$$

Using the product-type systems (57)-(72), the relations in (73), and the known fact that the difference equation

$$
\zeta_{n}=\zeta_{n-1}^{a_{1}} \zeta_{n-2}^{a_{2}} \cdots \zeta_{n-k}^{a_{k}}, \quad n \in \mathbb{N}_{0}
$$

where $a_{j} \in \mathbb{Z}, j=\overline{1, k}, a_{k} \neq 0$, is solvable, similarly as in the proof of Theorem 1 the following result can be proved. Because of the similarity the proof is omitted and left to the reader as an exercise. Note that this is not so unexpected since for the case when the initial values are positive by taking the logarithm, systems (57)-(72) are transformed to (15)-(30) (of course, it is not the proof in the general case, but instead of that the corresponding substitutions have to be used).

Theorem 2 Assume that $a \in \mathbb{C} \backslash\{0\}$. Then system of difference equations (11) is (theoretically) solvable.

\section{Case $k=0, I=1, a \neq 0$}

In this section we consider product-type systems (57)-(72) in the special case $k=0, l=1$, with $a \neq 0$, as a concrete example. We show that these systems are solvable in closed form, from which along with (73) will follow the solvability of systems (40)-(55) in the case.

First note that in the case the systems in (57)-(72) become

$$
\begin{array}{ll}
\zeta_{n+1}=\zeta_{n} \zeta_{n-1}, & \eta_{n+1}=\zeta_{n} \zeta_{n-1}, \\
\zeta_{n+1}=\zeta_{n} \zeta_{n-1}, & \eta_{n+1}=\eta_{n} \zeta_{n-1}, \\
\zeta_{n+1}=\zeta_{n} \zeta_{n-1}, & \eta_{n+1}=\zeta_{n} \eta_{n-1}, \\
\zeta_{n+1}=\zeta_{n} \zeta_{n-1}, & \eta_{n+1}=\eta_{n} \eta_{n-1}, \\
\zeta_{n+1}=\eta_{n} \zeta_{n-1}, & \eta_{n+1}=\zeta_{n} \zeta_{n-1}, \\
\zeta_{n+1}=\eta_{n} \zeta_{n-1}, & \eta_{n+1}=\eta_{n} \zeta_{n-1}, \\
\zeta_{n+1}=\eta_{n} \zeta_{n-1}, & \eta_{n+1}=\zeta_{n} \eta_{n-1}, \\
\zeta_{n+1}=\eta_{n} \zeta_{n-1}, & \eta_{n+1}=\eta_{n} \eta_{n-1}, \\
\zeta_{n+1}=\zeta_{n} \eta_{n-1}, & \eta_{n+1}=\zeta_{n} \zeta_{n-1}, \\
\zeta_{n+1}=\zeta_{n} \eta_{n-1}, & \eta_{n+1}=\eta_{n} \zeta_{n-1}, \\
\zeta_{n+1}=\zeta_{n} \eta_{n-1}, & \eta_{n+1}=\zeta_{n} \eta_{n-1}, \\
\zeta_{n+1}=\zeta_{n} \eta_{n-1}, & \eta_{n+1}=\eta_{n} \eta_{n-1}, \\
\zeta_{n+1}=\eta_{n} \eta_{n-1}, & \eta_{n+1}=\zeta_{n} \zeta_{n-1}, \\
\zeta_{n+1}=\eta_{n} \eta_{n-1}, & \eta_{n+1}=\eta_{n} \zeta_{n-1},
\end{array}
$$




$$
\begin{array}{ll}
\zeta_{n+1}=\eta_{n} \eta_{n-1}, & \eta_{n+1}=\zeta_{n} \eta_{n-1}, \\
\zeta_{n+1}=\eta_{n} \eta_{n-1}, & \eta_{n+1}=\eta_{n} \eta_{n-1},
\end{array}
$$

$n \in \mathbb{N}_{0}$, respectively.

Before we start considering systems (74)-(89), we formulate a known lemma which will be used in the rest of the paper.

Lemma 1 Let $x_{k}, k=\overline{1, m}$, be the distinct roots of the polynomial $p_{m}(x)=c_{m} x^{m}+\cdots+c_{1} x+$ $c_{0}$.

Then

$$
\sum_{k=1}^{m} \frac{x_{k}^{j}}{p_{m}^{\prime}\left(x_{k}\right)}=0
$$

when $0 \leq j \leq m-2$, and

$$
\sum_{k=1}^{l} \frac{x_{k}^{m-1}}{p_{m}^{\prime}\left(x_{k}\right)}=\frac{1}{c_{m}} .
$$

\subsection{Solution to system (74)}

First, note that from (74) it follows that

$$
\zeta_{n}=\eta_{n}, \quad n \in \mathbb{N} .
$$

The first equation in (74) is a special case of the difference equation

$$
\zeta_{n+1}=\zeta_{n}^{\alpha} \zeta_{n-1}^{\beta}, \quad n \in \mathbb{N}_{0}
$$

A solution to the equation can be found in [69] (see also [35, 40] or some of the methods used in the cases which follow), and its general solution is given by

$$
\zeta_{n}=\zeta_{0}^{f_{n+1}} \zeta_{-1}^{f_{n}}, \quad n \in \mathbb{N}_{0}
$$

where $\left(f_{n}\right)_{n \in \mathbb{N}_{0}}$ is the Fibonacci sequence.

Hence, we have

$$
\eta_{n}=\zeta_{0}^{f_{n+1}} \zeta_{-1}^{f_{n}}, \quad n \in \mathbb{N}
$$

Note also that formula (90) holds also for $n=-1$.

Corollary 1 Assume that $k=0, l=1$, and $a \in \mathbb{C} \backslash\{0\}$. Then the general solution to system (40) is given by

$$
\begin{aligned}
& x_{n}=\sqrt{a} \frac{\left(\frac{x_{0}+\sqrt{a}}{x_{0}-\sqrt{a}}\right)^{f_{n+1}}\left(\frac{x_{-1}+\sqrt{a}}{x_{-1}-\sqrt{a}}\right)^{f_{n}}+1}{\left(\frac{x_{0}+\sqrt{a}}{x_{0}-\sqrt{a}}\right) f_{n+1}\left(\frac{x_{-1}+\sqrt{a}}{x_{-1}-\sqrt{a}}\right)_{n}-1}, \quad n \geq-1, \\
& y_{n}=\sqrt{a} \frac{\left(\frac{x_{0}+\sqrt{a}}{x_{0}-\sqrt{a}}\right)^{f_{n+1}}\left(\frac{x_{-1}+\sqrt{a}}{x_{-1}-\sqrt{a}}\right)^{f_{n}}+1}{\left(\frac{x_{0}+\sqrt{a}}{x_{0}-\sqrt{a}}\right) f_{n+1}\left(\frac{x_{-1}+\sqrt{a}}{x_{-1}-\sqrt{a}}\right)^{f_{n}}-1}, \quad n \in \mathbb{N} .
\end{aligned}
$$




\subsection{Solution to system (75)}

First, note that from the previous case we have that formula (90) holds. Using the formula in the second equation in (75), it follows that

$$
\eta_{n+1}=\eta_{n} \zeta_{n-1}=\eta_{n} \zeta_{0}^{f_{n}} \zeta_{-1}^{f_{n-1}}, \quad n \in \mathbb{N}_{0}
$$

from which it follows that

$$
\begin{aligned}
\eta_{n} & =\eta_{1} \zeta_{0}^{\sum_{j=1}^{n-1} f_{j}} \zeta_{-1}^{\sum_{j=0}^{n-2} f_{j}} \\
& =\eta_{0} \zeta_{-1} \zeta_{0}^{\sum_{j=1}^{n-1}\left(f_{j+1}-f_{j-1}\right)} \zeta_{-1}^{\sum_{j=0}^{n-2}\left(f_{j+1}-f_{j-1}\right)} \\
& =\eta_{0} \zeta_{-1} \zeta_{0}^{f_{n}+f_{n-1}-f_{1}-f_{0}} \zeta_{-1}^{f_{n-1}+f_{n-2}-f_{0}-f_{-1}} \\
& =\eta_{0} \zeta_{0}^{f_{n+1}-1} \zeta_{-1}^{f_{n}}
\end{aligned}
$$

for $n \in \mathbb{N}_{0}$.

Formulas (90) and (93) are the closed-form formulas for general solution to system (75).

Corollary 2 Assume that $k=0, l=1$, and $a \in \mathbb{C} \backslash\{0\}$. Then the general solution to system (41) is given by

$$
\begin{aligned}
& x_{n}=\sqrt{a} \frac{\left(\frac{x_{0}+\sqrt{a}}{x_{0}-\sqrt{a}}\right)^{f_{n+1}\left(\frac{x_{-1}+\sqrt{a}}{x_{-1}-\sqrt{a}}\right) f_{n}+1}}{\left(\frac{x_{0}+\sqrt{a}}{x_{0}-\sqrt{a}}\right)_{n+1}\left(\frac{x_{-1}+\sqrt{a}}{x_{-1}-\sqrt{a}}\right) f_{n}-1}, \quad n \geq-1, \\
& y_{n}=\sqrt{a} \frac{\left(\frac{y_{0}+\sqrt{a}}{y_{0}-\sqrt{a}}\right)\left(\frac{x_{0}+\sqrt{a}}{x_{0}-\sqrt{a}}\right)^{f_{n+1}-1}\left(\frac{x_{-1}+\sqrt{a}}{x_{-1}-\sqrt{a}}\right)^{f_{n}}+1}{\left(\frac{y_{0}+\sqrt{a}}{y_{0}-\sqrt{a}}\right)\left(\frac{x_{0}+\sqrt{a}}{x_{0}-\sqrt{a}}\right)^{f_{n+1}-1}\left(\frac{x_{-1}+\sqrt{a}}{x_{-1}-\sqrt{a}}\right) f_{n}-1}, \quad n \in \mathbb{N}_{0} .
\end{aligned}
$$

\subsection{Solution to system (76)}

Clearly, formula (90) holds. Using the formula in the second equation in (76), we get

$$
\eta_{n+1}=\zeta_{n} \eta_{n-1}=\eta_{n-1} \zeta_{0}^{f_{n+1}} \zeta_{-1}^{f_{n}}, \quad n \in \mathbb{N}_{0}
$$

which is an equation with interlacing indices.

Hence, we have

$$
\eta_{2 n+i}=\eta_{2(n-1)+i} \zeta_{0}^{f_{2 n+i}} \zeta_{-1}^{f_{2 n+i-1}}
$$

for $n \in \mathbb{N}, i=-1,0$, from which it follows that

$$
\begin{aligned}
\eta_{2 n} & =\eta_{0} \zeta_{0}^{\sum_{j=1}^{n} f_{2 j}} \zeta_{-1}^{\sum_{j=1}^{n} f_{2 j-1}} \\
& =\eta_{0} \zeta_{0}^{\sum_{j=1}^{n}\left(f_{2 j+1}-f_{2 j-1}\right)} \zeta_{-1}^{\sum_{j=1}^{n}\left(f_{2 j}-f_{2 j-2}\right)} \\
& =\eta_{0} \zeta_{0}^{f_{2 n+1}-1} \zeta_{-1}^{f_{2 n}}
\end{aligned}
$$


for $n \in \mathbb{N}_{0}$, and

$$
\begin{aligned}
\eta_{2 n+1} & =\eta_{-1} \zeta_{0}^{\sum_{j=0}^{n} f_{2 j+1}} \zeta_{-1}^{\sum_{j=0}^{n} f_{2 j}} \\
& =\eta_{-1} \zeta_{0}^{\sum_{j=0}^{n}\left(f_{2 j+2}-f_{2 j}\right)} \zeta_{-1}^{\sum_{j=0}^{n}\left(f_{2 j+1}-f_{2 j-1}\right)} \\
& =\eta_{-1} \zeta_{0}^{f_{2 n+2}} \zeta_{-1}^{f_{2 n+1}-1}
\end{aligned}
$$

for $n \geq-1$.

Formulas (90), (96), and (97) are the closed-form formulas for general solution to system (76).

Corollary 3 Assume that $k=0, l=1$, and $a \in \mathbb{C} \backslash\{0\}$. Then the general solution to system (42) is given by

$$
\begin{aligned}
& x_{n}=\sqrt{a} \frac{\left(\frac{x_{0}+\sqrt{a}}{x_{0}-\sqrt{a}}\right)^{f_{n+1}}\left(\frac{x_{-1}+\sqrt{a}}{x_{-1}-\sqrt{a}}\right) f_{n}+1}{\left(\frac{x_{0}+\sqrt{a}}{x_{0}-\sqrt{a}}\right) f_{n+1}\left(\frac{x_{-1}+\sqrt{a}}{x_{-1}-\sqrt{a}}\right) f_{n}-1}, \quad n \geq-1, \\
& y_{2 n}=\sqrt{a} \frac{\left(\frac{y_{0}+\sqrt{a}}{y_{0}-\sqrt{a}}\right)\left(\frac{x_{0}+\sqrt{a}}{x_{0}-\sqrt{a}}\right)_{2 n+1}-1\left(\frac{x_{-1}+\sqrt{a}}{x_{-1}-\sqrt{a}}\right) f_{2 n}+1}{\left(\frac{y_{0}+\sqrt{a}}{y_{0}-\sqrt{a}}\right)\left(\frac{x_{0}+\sqrt{a}}{x_{0}-\sqrt{a}}\right) f_{2 n+1}-1\left(\frac{x_{-1}+\sqrt{a}}{x_{-1}-\sqrt{a}}\right) f_{2 n}-1}, \quad n \in \mathbb{N}_{0}, \\
& y_{2 n+1}=\sqrt{a} \frac{\left(\frac{y_{-1}+\sqrt{a}}{y_{-1}-\sqrt{a}}\right)\left(\frac{x_{0}+\sqrt{a}}{x_{0}-\sqrt{a}}\right)^{f_{2 n+2}}\left(\frac{x_{-1}+\sqrt{a}}{x_{-1}-\sqrt{a}}\right)^{f_{2 n+1}-1}+1}{\left(\frac{y_{-1}+\sqrt{a}}{y_{-1}-\sqrt{a}}\right)\left(\frac{x_{0}+\sqrt{a}}{x_{0}-\sqrt{a}}\right)^{f_{2 n+2}\left(\frac{x_{-1}+\sqrt{a}}{x_{-1}-\sqrt{a}}\right) f_{2 n+1}-1}, \quad n \geq-1 .}
\end{aligned}
$$

\subsection{Solution to system (77)}

Clearly, formula (90) holds, but since the second equation in (77) has the same form, it is clear that the solutions to the second equation in system (77) are obtained by replacing each letter $\zeta$ by $\eta$ in (90), that is, we have

$$
\eta_{n}=\eta_{0}^{f_{n+1}} \eta_{-1}^{f_{n}}, \quad n \geq-1 .
$$

Hence, formulas (90) and (98) are the closed-form formulas for general solution to system (77).

Corollary 4 Assume that $k=0, l=1$, and $a \in \mathbb{C} \backslash\{0\}$. Then the general solution to system (43) is given by

$$
\begin{array}{ll}
x_{n}=\sqrt{a} \frac{\left(\frac{x_{0}+\sqrt{a}}{x_{0}-\sqrt{a}}\right)^{f_{n+1}}\left(\frac{x_{-1}+\sqrt{a}}{x_{-1}-\sqrt{a}}\right)^{f_{n}}+1}{\left(\frac{x_{0}+\sqrt{a}}{x_{0}-\sqrt{a}}\right) f_{n+1}\left(\frac{x_{-1}+\sqrt{a}}{x_{-1}-\sqrt{a}}\right)_{n}-1}, & n \geq-1, \\
y_{n}=\sqrt{a} \frac{\left(\frac{y_{0}+\sqrt{a}}{y_{0}-\sqrt{a}}\right)^{f_{n+1}\left(\frac{y_{-1}+\sqrt{a}}{y_{-1}-\sqrt{a}}\right)^{f_{n}}+1}}{\left(\frac{y_{0}+\sqrt{a}}{y_{0}-\sqrt{a}}\right) f_{n+1}\left(\frac{y_{-1}+\sqrt{a}}{y_{-1}-\sqrt{a}}\right) f_{n}-1}, \quad n \geq-1 .
\end{array}
$$

\subsection{Solution to system (78)}

If we use the first equation in (78) into the second one, we get

$$
\zeta_{n+1}=\zeta_{n-1}^{2} \zeta_{n-2}
$$

for $n \in \mathbb{N}$. 
Let

$$
b_{0}=2, \quad c_{0}=1, \quad d_{0}=0 .
$$

Then equation (99) can be written as

$$
\zeta_{n+1}=\zeta_{n-1}^{b_{0}} \zeta_{n-2}^{c_{0}} \zeta_{n-3}^{d_{0}}=\zeta_{n-1}^{b_{0}} \zeta_{n-2}^{c_{0}}
$$

for $n \in \mathbb{N}$.

Using (101), where $n$ is replaced by $n-2$ into (101), we obtain

$$
\begin{aligned}
\zeta_{n+1} & =\zeta_{n-1}^{b_{0}} \zeta_{n-2}^{c_{0}} \zeta_{n-3}^{d_{0}} \\
& =\left(\zeta_{n-3}^{b_{0}} \zeta_{n-4}^{c_{0}}\right)^{b_{0}} \zeta_{n-2}^{c_{0}} \zeta_{n-3}^{d_{0}} \\
& =\zeta_{n-2}^{c_{0}} \zeta_{n-3}^{b_{0} b_{0}+d_{0}} \zeta_{n-4}^{c_{0} b_{0}} \\
& =\zeta_{n-2}^{b_{1}} \zeta_{n-3}^{c_{1}} \zeta_{n-4}^{d_{1}},
\end{aligned}
$$

where

$$
b_{1}:=c_{0}, \quad c_{1}:=b_{0} b_{0}+d_{0}, \quad d_{1}:=c_{0} b_{0} .
$$

Assume that we have proved

$$
\zeta_{n+1}=\zeta_{n-k}^{b_{k-1}} \zeta_{n-k-1}^{c_{k-1}} \zeta_{n-k-2}^{d_{k-1}}
$$

where

$$
b_{k-1}:=c_{k-2}, \quad c_{k-1}:=b_{0} b_{k-2}+d_{k-2}, \quad d_{k-1}:=c_{0} b_{k-2},
$$

for $k \geq 2$ and every $n \geq k+1$.

Using (101), where $n$ is replaced by $n-k-1$ into (104), we obtain

$$
\begin{aligned}
\zeta_{n+1} & =\zeta_{n-k}^{b_{k-1}} \zeta_{n-k-1}^{c_{k-1}} \zeta_{n-k-2}^{d_{k-1}} \\
& =\left(\zeta_{n-k-2}^{b_{0}} \zeta_{n-k-3}^{c_{0}}\right)^{b_{k-1}} \zeta_{n-k-1}^{c_{k-1}} \zeta_{n-k-2}^{d_{k-1}} \\
& =\zeta_{n-k-1}^{c_{k-1}} \zeta_{n-k-2}^{b_{0} b_{k-1}+d_{k-1}} \zeta_{n-k-3}^{c_{0} b_{k-1}} \\
& =\zeta_{n-k-1}^{b_{k}} \zeta_{n-k-2}^{c_{k}} \zeta_{n-k-3}^{d_{k}}
\end{aligned}
$$

where

$$
b_{k}:=c_{k-1}, \quad c_{k}:=b_{0} b_{k-1}+d_{k-1}, \quad d_{k}:=c_{0} b_{k-1} .
$$

From (102), (103), (106), (107), and the induction it follows that (104) and (105) hold for every $2 \leq k \leq n-1$. 
From (100) and (107), we have

$$
b_{k}:=c_{k-1}, \quad c_{k}:=2 b_{k-1}+d_{k-1}, \quad d_{k}:=b_{k-1} .
$$

If in (104) we replace $n$ by $n-1$, take $k=n-2$, and use (108), we have

$$
\begin{aligned}
\zeta_{n} & =\zeta_{1}^{b_{n-3}} \zeta_{0}^{c_{n-3}} \zeta_{-1}^{d_{n-3}} \\
& =\left(\eta_{0} \zeta_{-1}\right)^{b_{n-3}} \zeta_{0}^{c_{n-3}} \zeta_{-1}^{d_{n-3}} \\
& =\eta_{0}^{b_{n-3}} \zeta_{-1}^{b_{n-3}+d_{n-3}} \zeta_{0}^{c_{n-3}} \\
& =\eta_{0}^{b_{n-3}} \zeta_{-1}^{b_{n-3}+b_{n-4}} \zeta_{0}^{b_{n-2}}
\end{aligned}
$$

for $n \geq 4$.

From (108) it follows that

$$
b_{k}=2 b_{k-2}+b_{k-3}, \quad k \geq 3
$$

from which along with the first and third equation in (107) it follows that the sequences $\left(b_{k}\right)_{k \in \mathbb{N}_{0}},\left(c_{k}\right)_{k \in \mathbb{N}_{0}},\left(d_{k}\right)_{k \in \mathbb{N}_{0}}$ are solutions to the difference equation

$$
z_{k+3}-2 z_{k+1}-z_{k}=0, \quad k \in \mathbb{N}_{0}
$$

The associated characteristic polynomial to equation (110) is

$$
P_{3}(\lambda)=\lambda^{3}-2 \lambda-1=(\lambda+1)\left(\lambda^{2}-\lambda-1\right),
$$

from which it follows that $-1, \lambda_{1}$, and $\lambda_{2}$ are the characteristic zeros.

Hence, the general solution to (110) is given by

$$
b_{n}=\alpha_{1}(-1)^{n}+\alpha_{2} \lambda_{1}^{n}+\alpha_{3} \lambda_{2}^{n}
$$

Now note that from (108) it follows that $b_{1}=c_{0}=1, c_{1}=2 b_{0}+d_{0}=4, b_{2}=c_{1}=4$.

Hence, we should find the solution to equation (110) satisfying the following initial conditions:

$$
b_{0}=2, \quad b_{1}=1, \quad b_{2}=4
$$

that is, to find the constants $\alpha_{j}, j=\overline{1,3}$, such that

$$
\begin{aligned}
& \alpha_{1}+\alpha_{2}+\alpha_{3}=2, \\
& -\alpha_{1}+\alpha_{2} \lambda_{1}+\alpha_{3} \lambda_{2}=1, \\
& \alpha_{1}+\alpha_{2} \lambda_{1}^{2}+\alpha_{3} \lambda_{2}^{2}=4 .
\end{aligned}
$$

We have

$$
\alpha_{1}=\frac{\left|\begin{array}{ccc}
2 & 1 & 1 \\
1 & \lambda_{1} & \lambda_{2} \\
4 & \lambda_{1}^{2} & \lambda_{2}^{2}
\end{array}\right|}{\Delta}=\frac{\lambda_{2}-\lambda_{1}}{\lambda_{2}-\lambda_{1}}=1,
$$




$$
\begin{aligned}
& \alpha_{2}=\frac{\left|\begin{array}{ccc}
1 & 2 & 1 \\
-1 & 1 & \lambda_{2} \\
1 & 4 & \lambda_{2}^{2}
\end{array}\right|}{\Delta}=\frac{3+\sqrt{5}}{2 \sqrt{5}}=\frac{\lambda_{1}^{2}}{\sqrt{5}}=\frac{\lambda_{1}^{2}}{\lambda_{1}-\lambda_{2}}, \\
& \alpha_{3}=\frac{\left|\begin{array}{ccc}
1 & 1 & 2 \\
-1 & \lambda_{1} & 1 \\
1 & \lambda_{1}^{2} & 4
\end{array}\right|}{\Delta}=\frac{\sqrt{5}-3}{2 \sqrt{5}}=-\frac{\lambda_{2}^{2}}{\sqrt{5}}=-=\frac{\lambda_{2}^{2}}{\lambda_{2}-\lambda_{1}},
\end{aligned}
$$

where

$$
\Delta=\left|\begin{array}{ccc}
1 & 1 & 1 \\
-1 & \lambda_{1} & \lambda_{2} \\
1 & \lambda_{1}^{2} & \lambda_{2}^{2}
\end{array}\right|=\lambda_{2}-\lambda_{1}=-\sqrt{5}
$$

Using these formulas in (111), we obtain

$$
b_{n}=(-1)^{n}+\frac{\lambda_{1}^{n+2}-\lambda_{2}^{n+2}}{\sqrt{5}}=(-1)^{n}+f_{n+2} .
$$

Employing (114) in (109), it follows that

$$
\zeta_{n}=\eta_{0}^{(-1)^{n-1}+f_{n-1}} \zeta_{0}^{(-1)^{n}+f_{n}} \zeta_{-1}^{f_{n}}, \quad n \geq-1 .
$$

Using (115) in the second equation in (78), we obtain

$$
\begin{aligned}
\eta_{n} & =\zeta_{n-1} \zeta_{n-2} \\
& =\eta_{0}^{(-1)^{n}+f_{n-2}} \zeta_{0}^{(-1)^{n-1}+f_{n-1}} \zeta_{-1}^{f_{n-1}} \eta_{0}^{(-1)^{n-1}+f_{n-3}} \zeta_{0}^{(-1)^{n}+f_{n-2}} \zeta_{-1}^{f_{n-2}} \\
& =\eta_{0}^{f_{n-1}} \zeta_{0}^{f_{n}} \zeta_{-1}^{f_{n}}, \quad n \in \mathbb{N}_{0} .
\end{aligned}
$$

Formulas (115) and (116) are the closed-form formulas for general solution to system (78).

Corollary 5 Assume that $k=0, l=1$, and $a \in \mathbb{C} \backslash\{0\}$. Then the general solution to system (44) is given by

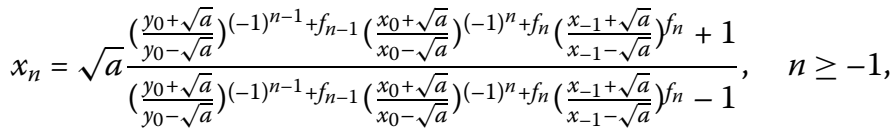

$$
\begin{aligned}
& y_{n}=\sqrt{a} \frac{\left(\frac{y_{0}+\sqrt{a}}{y_{0}-\sqrt{a}}\right)^{f_{n-1}}\left(\frac{x_{0}+\sqrt{a}}{x_{0}-\sqrt{a}}\right)^{f_{n}}\left(\frac{x_{-1}+\sqrt{a}}{x_{-1}-\sqrt{a}}\right)^{f_{n}}+1}{\left(\frac{y_{0}+\sqrt{a}}{y_{0}-\sqrt{a}}\right) f_{n-1}\left(\frac{x_{0}+\sqrt{a}}{x_{0}-\sqrt{a}}\right) f_{n}\left(\frac{x_{-1}+\sqrt{a}}{x_{-1}-\sqrt{a}}\right) f_{n}-1}, \quad n \in \mathbb{N}_{0} .
\end{aligned}
$$

\subsection{Solution to system (79)}

From the equations in (79) we see that $\zeta_{n}=\eta_{n}, n \in \mathbb{N}$. Hence, $\zeta_{n+1}=\zeta_{n} \zeta_{n-1}, n \geq 2$.

From this and (90), we have

$$
\begin{aligned}
\zeta_{n} & =\zeta_{2}^{f_{n-1}} \zeta_{1}^{f_{n-2}} \\
& =\left(\eta_{0} \zeta_{-1} \zeta_{0}\right)^{f_{n-1}}\left(\eta_{0} \zeta_{-1}\right)^{f_{n-2}} \\
& =\eta_{0}^{f_{n}} \zeta_{-1}^{f_{n}} \zeta_{0}^{f_{n-1}}, \quad n \in \mathbb{N}_{0}
\end{aligned}
$$


and consequently

$$
\eta_{n}=\eta_{0}^{f_{n}} \zeta_{-1}^{f_{n}} \zeta_{0}^{f_{n-1}}, \quad n \in \mathbb{N} .
$$

Formulas (117) and (118) are the closed-form formulas for general solution to system (79).

Corollary 6 Assume that $k=0, l=1$, and $a \in \mathbb{C} \backslash\{0\}$. Then the general solution to system (45) is given by

$$
\begin{aligned}
& x_{n}=\sqrt{a} \frac{\left(\frac{y_{0}+\sqrt{a}}{y_{0}-\sqrt{a}}\right)_{n}\left(\frac{x_{0}+\sqrt{a}}{x_{0}-\sqrt{a}}\right) f_{n-1}\left(\frac{x_{-1}+\sqrt{a}}{x_{-1}-\sqrt{a}}\right) f_{n}+1}{\left(\frac{y_{0}+\sqrt{a}}{y_{0}-\sqrt{a}}\right) f_{n}\left(\frac{x_{0}+\sqrt{a}}{x_{0}-\sqrt{a}}\right) f_{n-1}\left(\frac{x_{-1}+\sqrt{a}}{x_{-1}-\sqrt{a}}\right) f_{n}-1}, \quad n \in \mathbb{N}_{0}, \\
& y_{n}=\sqrt{a} \frac{\left(\frac{y_{0}+\sqrt{a}}{y_{0}-\sqrt{a}}\right) f_{n}\left(\frac{x_{0}+\sqrt{a}}{x_{0}-\sqrt{a}}\right) f_{n-1}\left(\frac{x_{-1}+\sqrt{a}}{x_{1}-\sqrt{a}}\right) f_{n}+1}{\left(\frac{y_{0}+\sqrt{a}}{y_{0}-\sqrt{a}}\right) f_{n}\left(\frac{x_{0}+\sqrt{a}}{x_{0}-\sqrt{a}}\right) f_{n-1}\left(\frac{x_{-1}+\sqrt{a}}{x_{-1}-\sqrt{a}}\right) f_{n}-1}, \quad n \in \mathbb{N} .
\end{aligned}
$$

\subsection{Solution to system (80)}

Using the first equation in (80) into the second one, we get

$$
\zeta_{n+2}=\zeta_{n}^{3} \zeta_{n-2}^{-1}, \quad n \in \mathbb{N}
$$

from which it follows that the sequences $\left(\zeta_{2 n-1}\right)_{n \in \mathbb{N}_{0}}$ and $\left(\zeta_{2 n}\right)_{n \in \mathbb{N}_{0}}$ satisfy the difference equation

$$
z_{n}=z_{n-1}^{3} z_{n-2}^{-1}, \quad n \geq 2
$$

(equation (119) is with interlacing indices; for the notion, see, e.g., [15]).

Let

$$
a_{0}=3 \quad \text { and } \quad b_{0}=-1 .
$$

Then

$$
\begin{aligned}
z_{n} & =z_{n-1}^{a_{0}} z_{n-2}^{b_{0}} \\
& =\left(z_{n-2}^{a_{0}} z_{n-3}^{b_{0}}\right)^{a_{0}} z_{n-2}^{b_{0}} \\
& =z_{n-2}^{a_{0} a_{0}+b_{0}} z_{n-3}^{b_{0} a_{0}} \\
& =z_{n-2}^{a_{1}} z_{n-3}^{b_{1}}
\end{aligned}
$$

for $n \geq 3$, where

$$
a_{1}:=a_{0} a_{0}+b_{0}, \quad b_{1}:=b_{0} a_{0} .
$$

Assume that we have proved

$$
z_{n}=z_{n-k-1}^{a_{k}} z_{n-k-2}^{b_{k}}
$$


for some $k \in \mathbb{N}$ and every $n \geq k+2$, and that

$$
a_{k}=a_{0} a_{k-1}+b_{k-1}, \quad b_{k}=b_{0} a_{k-1} .
$$

Then we have

$$
\begin{aligned}
z_{n} & =z_{n-k-1}^{a_{k}} z_{n-k-2}^{b_{k}} \\
& =\left(z_{n-k-2}^{a_{0}} z_{n-k-3}^{b_{0}}\right)^{a_{k}} z_{n-k-2}^{b_{k}} \\
& =z_{n-k-2}^{a_{0} a_{k}+b_{k}} z_{n-k-3}^{b_{0} a_{k}} \\
& =z_{n-k-2}^{a_{k+1}} z_{n-k-3}^{b_{k+1}}
\end{aligned}
$$

for $n \geq k+3$, where

$$
a_{k+1}:=a_{0} a_{k}+b_{k}, \quad b_{k+1}:=b_{0} a_{k} .
$$

From (122), (123), (126), (127), and the induction it follows that (124) and (125) hold for every $k, n \in \mathbb{N}$ such that $1 \leq k \leq n-2$.

If in (124) we take $k=n-2$, we get

$$
z_{n}=z_{1}^{a_{n-2}} z_{0}^{b_{n-2}}, \quad n \geq 2 .
$$

From (121) and (127) we see that the sequences $\left(a_{k}\right)_{k \in \mathbb{N}_{0}}$ and $\left(b_{k}\right)_{k \in \mathbb{N}_{0}}$ are solutions of the following difference equation:

$$
w_{k}-3 w_{k-1}+w_{k-2}=0, \quad k \geq 2 .
$$

The roots of the characteristic polynomial $P_{2}(t)=t^{2}-3 t+1$ associated with equation (129) are

$$
t_{1}=\frac{3+\sqrt{5}}{2}=\lambda_{1}^{2} \text { and } t_{2}=\frac{3-\sqrt{5}}{2}=\lambda_{2}^{2}
$$

where $\lambda_{1,2}$ are defined in (10).

The solution to equation (129) with initial values $w_{0}$ and $w_{1}$ is directly obtained from the de Moivre formula

$$
w_{k}=\frac{\left(w_{1}-t_{1} w_{0}\right) t_{2}^{k}-\left(w_{1}-t_{2} w_{0}\right) t_{1}^{k}}{t_{2}-t_{1}}
$$

for $k \in \mathbb{N}_{0}$ [3].

From (130) and (131), we have

$$
\begin{aligned}
a_{n} & =\frac{\left(a_{1}-t_{1} a_{0}\right) t_{2}^{n}-\left(a_{1}-t_{2} a_{0}\right) t_{1}^{n}}{t_{2}-t_{1}} \\
& =\frac{\left(a_{1}-\lambda_{1}^{2} a_{0}\right) \lambda_{2}^{2 n}-\left(a_{1}-\lambda_{2}^{2} a_{0}\right) \lambda_{1}^{2 n}}{\lambda_{2}-\lambda_{1}} \\
& =a_{1} f_{2 n}-a_{0} f_{2 n-2}
\end{aligned}
$$


for every $n \in \mathbb{N}_{0}$, from which along with the second equation in (125) it follows that

$$
b_{n}=b_{0} a_{n-1}=b_{0}\left(a_{1} f_{2 n-2}-a_{0} f_{2 n-4}\right)
$$

for $n \in \mathbb{N}_{0}$.

From (132), (133), and since $a_{0}=3$ and $a_{1}=a_{0}^{2}+b_{0}=8$, it follows that

$$
a_{n}=8 f_{2 n}-3 f_{2 n-2}, \quad n \in \mathbb{N}_{0},
$$

and

$$
b_{n}=-8 f_{2 n-2}+3 f_{2 n-4}, \quad n \in \mathbb{N}_{0} .
$$

Using formulas (134) and (135) in relation (128), we obtain

$$
z_{n}=z_{1}^{8 f_{2 n-4}-3 f_{2 n-6}} z_{0}^{-8 f_{2 n-6}+3 f_{2 n-8}}
$$

for $n \in \mathbb{N}_{0}$.

From (136) it follows that

$$
\begin{aligned}
\zeta_{2 n-1} & =\zeta_{1}^{8 f_{2 n-4}-3 f_{2 n-6}} \zeta_{-1}^{-8 f_{2 n-6}+3 f_{2 n-8}} \\
& =\left(\eta_{0} \zeta_{-1}\right)^{8 f_{2 n-4}-3 f_{2 n-6}} \zeta_{-1}^{-8 f_{2 n-6}+3 f_{2 n-8}} \\
& =\eta_{0}^{8 f_{2 n-4}-3 f_{2 n-6}} \zeta_{-1}^{8\left(f_{2 n-4}-f_{2 n-6}\right)-3\left(f_{2 n-6}-f_{2 n-8}\right)} \\
& =\eta_{0}^{8 f_{2 n-4}-3 f_{2 n-6}} \zeta_{-1}^{8 f_{2 n-5}-3 f_{2 n-7}}, \quad n \in \mathbb{N}_{0}
\end{aligned}
$$

and

$$
\begin{aligned}
\zeta_{2 n} & =\zeta_{2}^{8 f_{2 n-4}-3 f_{2 n-6}} \zeta_{0}^{-8 f_{2 n-6}+3 f_{2 n-8}} \\
& =\left(\zeta_{0}^{2} \eta_{-1}\right)^{8 f_{2 n-4}-3 f_{2 n-6}} \zeta_{0}^{-8 f_{2 n-6}+3 f_{2 n-8}} \\
& =\zeta_{0}^{8\left(2 f_{2 n-4}-f_{2 n-6}\right)-3\left(2 f_{2 n-6}-f_{2 n-8}\right)} \eta_{-1}^{8 f_{2 n-4}-3 f_{2 n-6}} \\
& =\zeta_{0}^{8 f_{2 n-3}-3 f_{2 n-5}} \eta_{-1}^{8 f_{2 n-4}-3 f_{2 n-6}}, \quad n \in \mathbb{N}_{0} .
\end{aligned}
$$

Further, we have

$$
\begin{aligned}
8 f_{k}-3 f_{k-2} & =5 f_{k}+3\left(f_{k}-f_{k-2}\right)=5 f_{k}+3 f_{k-1} \\
& =2 f_{k}+3\left(f_{k}+f_{k-1}\right)=2 f_{k}+3 f_{k+1} \\
& =2\left(f_{k}+f_{k+1}\right)+f_{k+1}=2 f_{k+2}+f_{k+1} \\
& =f_{k+2}+\left(f_{k+2}+f_{k+1}\right)=f_{k+2}+f_{k+3} \\
& =f_{k+4} .
\end{aligned}
$$

By using (139) in (137) and (138), we obtain

$$
\zeta_{2 n-1}=\eta_{0}^{f_{2 n}} \zeta_{-1}^{f_{2 n-1}}, \quad n \in \mathbb{N}_{0}
$$


and

$$
\zeta_{2 n}=\zeta_{0}^{f_{2 n+1}} \eta_{-1}^{f_{2 n}}, \quad n \in \mathbb{N}_{0}
$$

Using (140) and (141) in the relation $\eta_{n}=\zeta_{n+1} / \zeta_{n-1}$, we obtain

$$
\eta_{2 n-1}=\zeta_{2 n} / \zeta_{2 n-2}=\zeta_{0}^{f_{2 n+1}-f_{2 n-1}} \eta_{-1}^{f_{2 n}-f_{2 n-2}}=\zeta_{0}^{f_{2 n}} \eta_{-1}^{f_{2 n-1}}, \quad n \in \mathbb{N}
$$

(the last equality holds also for $n=0$ ) and

$$
\eta_{2 n}=\zeta_{2 n+1} / \zeta_{2 n-1}=\eta_{0}^{f_{2 n+2}-f_{2 n}} \zeta_{-1}^{f_{2 n+1}-f_{2 n-1}}=\eta_{0}^{f_{2 n+1}} \zeta_{-1}^{f_{2 n}}, \quad n \in \mathbb{N}_{0}
$$

Formulas (140)-(143) are the closed-form formulas for general solution to system (80).

Corollary 7 Assume that $k=0, l=1$, and $a \in \mathbb{C} \backslash\{0\}$. Then the general solution to system (46) is given by

$$
\begin{aligned}
& x_{2 n-1}=\sqrt{a} \frac{\left(\frac{y_{0}+\sqrt{a}}{y_{0}-\sqrt{a}}\right)^{2 n}\left(\frac{x_{-1}+\sqrt{a}}{x_{-1}-\sqrt{a}}\right) f_{2 n-1}+1}{\left(\frac{y_{0}+\sqrt{a}}{y_{0}-\sqrt{a}}\right) f_{2 n}\left(\frac{x_{-1}+\sqrt{a}}{x_{-1}-\sqrt{a}}\right) f_{2 n-1}-1}, \quad n \in \mathbb{N}_{0},
\end{aligned}
$$

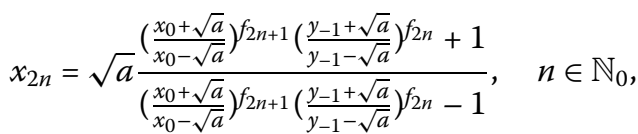

$$
\begin{aligned}
& y_{2 n-1}=\sqrt{a} \frac{\left(\frac{x_{0}+\sqrt{a}}{x_{0}-\sqrt{a}}\right)^{2 n}\left(\frac{y_{-1}+\sqrt{a}}{y_{-1}-\sqrt{a}}\right)^{f_{2 n-1}+1}}{\left(\frac{x_{0}+\sqrt{a}}{x_{0}-\sqrt{a}}\right) f_{2 n}\left(\frac{y_{-1}+\sqrt{a}}{y_{-1}-\sqrt{a}}\right) f_{2 n-1}-1}, \quad n \in \mathbb{N}_{0} \text {, }
\end{aligned}
$$

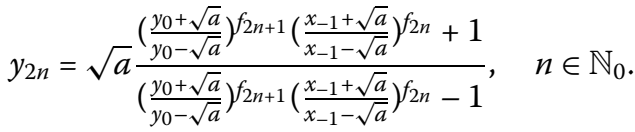

\subsection{Solution to system (81)}

This system is obtained from system (76) by interchanging letters $\zeta$ and $\eta$. Hence, its general solution is given by

$$
\begin{aligned}
& \eta_{n}=\eta_{0}^{f_{n+1}} \eta_{-1}^{f_{n}}, \quad n \geq-1, \\
& \zeta_{2 n}=\zeta_{0} \eta_{0}^{f_{2 n+1}-1} \eta_{-1}^{f_{2 n}}, \quad n \in \mathbb{N}_{0},
\end{aligned}
$$

and

$$
\zeta_{2 n+1}=\zeta_{-1} \eta_{0}^{f_{2 n+2}} \eta_{-1}^{f_{2 n+1}-1}, \quad n \geq-1
$$

Corollary 8 Assume that $k=0, l=1$, and $a \in \mathbb{C} \backslash\{0\}$. Then the general solution to system (47) is given by

$$
x_{2 n}=\sqrt{a} \frac{\left(\frac{x_{0}+\sqrt{a}}{x_{0}-\sqrt{a}}\right)\left(\frac{y_{0}+\sqrt{a}}{y_{0}-\sqrt{a}}\right)^{f_{2 n+1}-1}\left(\frac{y_{-1}+\sqrt{a}}{y_{-1}-\sqrt{a}}\right)^{f_{2 n}}+1}{\left(\frac{x_{0}+\sqrt{a}}{x_{0}-\sqrt{a}}\right)\left(\frac{y_{0}+\sqrt{a}}{y_{0}-\sqrt{a}}\right)^{f_{2 n+1}-1}\left(\frac{y_{-1}+\sqrt{a}}{y_{-1}-\sqrt{a}}\right) f_{2 n}-1}, \quad n \in \mathbb{N}_{0},
$$




$$
\begin{aligned}
& x_{2 n+1}=\sqrt{a} \frac{\left(\frac{x_{-1}+\sqrt{a}}{x_{-1}-\sqrt{a}}\right)\left(\frac{y_{0}+\sqrt{a}}{y_{0}-\sqrt{a}}\right)^{f_{2 n+2}\left(\frac{y_{-1}+\sqrt{a}}{y_{-1}-\sqrt{a}}\right)^{f_{2 n+1}-1}+1}}{\left.\left(\frac{x_{-1}+\sqrt{a}}{x_{-1}-\sqrt{a}}\right)\left(\frac{y_{0}+\sqrt{a}}{y_{0}-\sqrt{a}}\right)^{f_{2 n+2}\left(\frac{y_{-1}+\sqrt{a}}{y_{-1}-\sqrt{a}}\right.}\right)^{f_{2 n+1}-1}-1}, \quad n \geq-1, \\
& y_{n}=\sqrt{a} \frac{\left(\frac{y_{0}+\sqrt{a}}{y_{0}-\sqrt{a}}\right)^{f_{n+1}\left(\frac{y_{-1}+\sqrt{a}}{y_{-1}-\sqrt{a}}\right)^{f_{n}}+1}}{\left(\frac{y_{0}+\sqrt{a}}{y_{0}-\sqrt{a}}\right)^{f_{n+1}\left(\frac{y_{-1}+\sqrt{a}}{y_{-1}-\sqrt{a}}\right)^{f_{n}}-1}, \quad n \geq-1 .}
\end{aligned}
$$

\subsection{Solution to system (82)}

By using the second equation in (82) into the first one, we get

$$
\zeta_{n}=\zeta_{n-1} \zeta_{n-3} \zeta_{n-4}, \quad n \geq 3
$$

To solve equation (147) we use here a method that we have used in some of our previous papers (see, e.g., [37, 41]).

Let

$$
a_{0}=1, \quad b_{0}=0, \quad c_{0}=1, \quad d_{0}=1 .
$$

Then equation (147) can be written as follows:

$$
\zeta_{n}=\zeta_{n-1}^{a_{0}} \zeta_{n-2}^{b_{0}} \zeta_{n-3}^{c_{0}} \zeta_{n-4}^{d_{0}}, \quad n \geq 3
$$

From (149), we get

$$
\begin{aligned}
\zeta_{n} & =\zeta_{n-1}^{a_{0}} \zeta_{n-2}^{b_{0}} \zeta_{n-3}^{c_{0}} \zeta_{n-4}^{d_{0}} \\
& =\left(\zeta_{n-2}^{a_{0}} \zeta_{n-3}^{b_{0}} \zeta_{n-4}^{c_{0}} \zeta_{n-5}^{d_{0}}\right)^{a_{0}} \zeta_{n-2}^{b_{0}} \zeta_{n-3}^{c_{0}} \zeta_{n-4}^{d_{0}} \\
& =\zeta_{n-2}^{a_{0} a_{0}+b_{0}} \zeta_{n-3}^{b_{0} a_{0}+c_{0}} \zeta_{n-4}^{c_{0} a_{0}+d_{0}} \zeta_{n-5}^{d_{0} a_{0}} \\
& =\zeta_{n-2}^{a_{1}} \zeta_{n-3}^{b_{1}} \zeta_{n-4}^{c_{1}} \zeta_{n-5}^{d_{1}}
\end{aligned}
$$

for $n \geq 4$, where

$$
a_{1}:=a_{0} a_{0}+b_{0}, \quad b_{1}:=b_{0} a_{0}+c_{0}, \quad c_{1}:=c_{0} a_{0}+d_{0}, \quad d_{1}:=d_{0} a_{0} .
$$

Assume that we have proved

$$
\zeta_{n}=\zeta_{n-k-1}^{a_{k}} \zeta_{n-k-2}^{b_{k}} \zeta_{n-k-3}^{c_{k}} \zeta_{n-k-4}^{d_{k}}
$$

for some $k \in \mathbb{N}$ and every $n \geq k+3$, and that

$$
\begin{array}{ll}
a_{k}=a_{0} a_{k-1}+b_{k-1}, & b_{k}=b_{0} a_{k-1}+c_{k-1}, \\
c_{k}=c_{0} a_{k-1}+d_{k-1}, & d_{k}=d_{0} a_{k-1} .
\end{array}
$$


Then we have

$$
\begin{aligned}
\zeta_{n} & =\zeta_{n-k-1}^{a_{k}} \zeta_{n-k-2}^{b_{k}} \zeta_{n-k-3}^{c_{k}} \zeta_{n-k-4}^{d_{k}} \\
& =\left(\zeta_{n-k-2}^{a_{0}} \zeta_{n-k-3}^{b_{0}} \zeta_{n-k-4}^{c_{0}} \zeta_{n-k-5}^{d_{0}}\right)^{a_{k}} \zeta_{n-k-2}^{b_{k}} \zeta_{n-k-3}^{c_{k}} \zeta_{n-k-4}^{d_{k}} \\
& =\zeta_{n-k-2}^{a_{0} a_{k}+b_{k}} \zeta_{n-k-3}^{b_{0} a_{k}+c_{k}} \zeta_{n-k-4}^{c_{0} a_{k}+d_{k}} \zeta_{n-k-5}^{d_{0} a_{k}} \\
& =\zeta_{n-k-2}^{a_{k+1}} \zeta_{n-k-3}^{b_{k+1}} \zeta_{n-k-4}^{c_{k+1}} \zeta_{n-k-5}^{d_{k+1}}
\end{aligned}
$$

for $n \geq k+4$, where

$$
a_{k+1}:=a_{0} a_{k}+b_{k}, \quad b_{k+1}:=b_{0} a_{k}+c_{k}, \quad c_{k+1}:=c_{0} a_{k}+d_{k}, \quad d_{k+1}:=d_{0} a_{k} .
$$

From (150), (151), (154), (155), and the induction it follows that (152) and (153) hold for every $k, n \in \mathbb{N}$ such that $1 \leq k \leq n-3$.

From (148) and (155), we have

$$
a_{k+1}:=a_{k}+b_{k}, \quad b_{k+1}:=c_{k}, \quad c_{k+1}:=a_{k}+d_{k}, \quad d_{k+1}:=a_{k} .
$$

If in (152) we take $k=n-3$, we get

$$
\begin{aligned}
\zeta_{n} & =\zeta_{2}^{a_{n-3}} \zeta_{1}^{b_{n-3}} \zeta_{0}^{c_{n-3}} \zeta_{-1}^{d_{n-3}} \\
& =\left(\zeta_{0} \eta_{-1} \eta_{0}\right)^{a_{n-3}}\left(\zeta_{0} \eta_{-1}\right)^{b_{n-3}} \zeta_{0}^{c_{n-3}} \zeta_{-1}^{d_{n-3}} \\
& =\zeta_{-1}^{d_{n-3}} \zeta_{0}^{a_{n-3}+b_{n-3}+c_{n-3}} \eta_{-1}^{a_{n-3}+b_{n-3}} \eta_{0}^{a_{n-3}} \\
& =\zeta_{-1}^{a_{n-4}} \zeta_{0}^{a_{n-1}} \eta_{-1}^{a_{n-2}} \eta_{0}^{a_{n-3}}
\end{aligned}
$$

for $n \geq 3$.

Using (157) into the second equation in (82), we obtain

$$
\begin{aligned}
\eta_{n} & =\zeta_{n-1} \zeta_{n-2}=\zeta_{-1}^{a_{n-5}} \zeta_{0}^{a_{n-2}} \eta_{-1}^{a_{n-3}} \eta_{0}^{a_{n-4}} \zeta_{-1}^{a_{n-6}} \zeta_{0}^{a_{n-3}} \eta_{-1}^{a_{n-4}} \eta_{0}^{a_{n-5}} \\
& =\zeta_{-1}^{a_{n-5}+a_{n-6}} \zeta_{0}^{a_{n-2}+a_{n-3}} \eta_{-1}^{a_{n-3}+a_{n-4}} \eta_{0}^{a_{n-4}+a_{n-5}} .
\end{aligned}
$$

From (153) we see that the sequences $\left(a_{k}\right)_{k \in \mathbb{N}_{0}},\left(b_{k}\right)_{k \in \mathbb{N}_{0}},\left(c_{k}\right)_{k \in \mathbb{N}_{0}}$, and $\left(d_{k}\right)_{k \in \mathbb{N}_{0}}$ are solutions to the following difference equation:

$$
w_{k}-w_{k-1}-w_{k-3}-w_{k-4}=0, \quad k \geq 4 .
$$

The roots of the characteristic polynomial

$$
P_{4}(t)=t^{4}-t^{3}-t-1=\left(t^{2}-t-1\right)\left(t^{2}+1\right)
$$

associated with equation (159) are

$$
t_{1}=i, \quad t_{2}=-i, \quad t_{3}=\lambda_{1}, \quad t_{4}=\lambda_{2} .
$$


The general solution to equation (159) is

$$
w_{k}=\alpha_{1} i^{k}+\alpha_{2}(-i)^{k}+\alpha_{3} \lambda_{1}^{k}+\alpha_{4} \lambda_{2}^{k}, \quad k \in \mathbb{N}_{0} .
$$

From (148) and (156) with $k=0,-1,-2,-3$, by some simple calculations, it follows that (see, e.g., [36, 39])

$$
\begin{array}{llll}
a_{-1}=1, & b_{-1}=0, & c_{-1}=0, & d_{-1}=0, \\
a_{-2}=0, & b_{-2}=1, & c_{-2}=0, & d_{-2}=0, \\
a_{-3}=0, & b_{-3}=0, & c_{-3}=1, & d_{-3}=0, \\
a_{-4}=0, & b_{-4}=0, & c_{-4}=0, & d_{-4}=1 .
\end{array}
$$

From (148), (162), and Lemma 1, it follows that

$$
a_{n}=\frac{i^{n+4}}{P_{4}^{\prime}(i)}+\frac{(-i)^{n+4}}{P_{4}^{\prime}(-i)}+\frac{\lambda_{1}^{n+4}}{P_{4}^{\prime}\left(\lambda_{1}\right)}+\frac{\lambda_{2}^{n+4}}{P_{4}^{\prime}\left(\lambda_{2}\right)}, \quad n \geq-4 .
$$

From this and since

$$
\begin{aligned}
& P_{4}^{\prime}(i)=(i-(-i))\left(i-\lambda_{1}\right)\left(i-\lambda_{2}\right)=2(1-2 i), \\
& P_{4}^{\prime}(-i)=(-i-i)\left(-i-\lambda_{1}\right)\left(-i-\lambda_{2}\right)=2(1+2 i), \\
& P_{4}^{\prime}\left(\lambda_{1}\right)=\left(\lambda_{1}-i\right)\left(\lambda_{1}-(-i)\right)\left(\lambda_{1}-\lambda_{2}\right)=\sqrt{5} \lambda_{1}\left(\lambda_{1}-\lambda_{2}\right)=5 \lambda_{1}, \\
& P_{4}^{\prime}\left(\lambda_{2}\right)=\left(\lambda_{2}-i\right)\left(\lambda_{2}-(-i)\right)\left(\lambda_{2}-\lambda_{1}\right)=\sqrt{5} \lambda_{2}\left(\lambda_{1}-\lambda_{2}\right)=5 \lambda_{2},
\end{aligned}
$$

and after some calculation, we have

$$
\begin{aligned}
a_{n} & =\frac{(1+2 i) i^{n}}{10}+\frac{(1-2 i)(-i)^{n}}{10}+\frac{\lambda_{1}^{n+3}+\lambda_{2}^{n+3}}{5} \\
& =\frac{\operatorname{Re}\left((1+2 i) i^{n}\right)+\lambda_{1}^{n+3}+\lambda_{2}^{n+3}}{5}, \quad n \geq-4 .
\end{aligned}
$$

Using (164) in (157), we obtain

$$
\begin{aligned}
\zeta_{n}= & \zeta_{-1}^{\frac{\operatorname{Re}\left((1+2 i) i^{n}\right)+\lambda_{1}^{n-1}+\lambda_{2}^{n-1}}{5}} \zeta_{0}^{\frac{\operatorname{Re}\left((1+2 i) i^{n-1}\right)+\lambda_{1}^{n+2}+\lambda_{2}^{n+2}}{5}} \\
& \times \eta_{-1}^{\frac{\operatorname{Re}\left((1+2 i) i^{n-2}\right)+\lambda_{1}^{n+1}+\lambda_{2}^{n+1}}{5}} \eta_{0}^{\frac{\operatorname{Re}\left((1+2 i) i^{n-3}\right)+\lambda_{1}^{n}+\lambda_{2}^{n}}{5}}, \quad n \geq-1 .
\end{aligned}
$$

By some simple calculation, we have

$$
a_{n}+a_{n-1}=\frac{\operatorname{Re}\left((3+i) i^{n}\right)+\lambda_{1}^{n+4}+\lambda_{2}^{n+4}}{5}, \quad n \geq-3 .
$$

Using (166) into (158), we have

$$
\begin{aligned}
\eta_{n}= & \zeta_{-1} \frac{\operatorname{Re}\left((3+i) i^{n-1}\right)+\lambda_{1}^{n-1}+\lambda_{2}^{n-1}}{5} \zeta_{0}^{\frac{\operatorname{Re}\left((3+i) i^{n-2}\right)+\lambda_{1}^{n+2}+\lambda_{2}^{n+2}}{5}} \\
& \times \eta_{-1}^{\frac{\operatorname{Re}\left((3+i) i^{n-3}\right)+\lambda_{1}^{n+1}+\lambda_{2}^{n+1}}{5}} \eta_{0}^{\frac{\operatorname{Re}\left((3+i) i^{n}\right)+\lambda_{1}^{n}+\lambda_{2}^{n}}{5}} .
\end{aligned}
$$


Corollary 9 Assume that $k=0, l=1$, and $a \in \mathbb{C} \backslash\{0\}$. Then the general solution to system (48) is given by

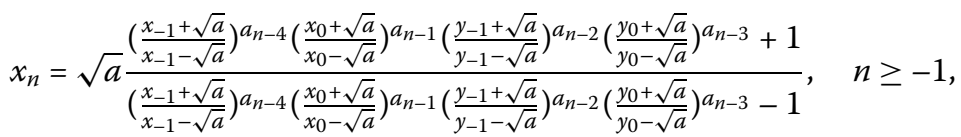

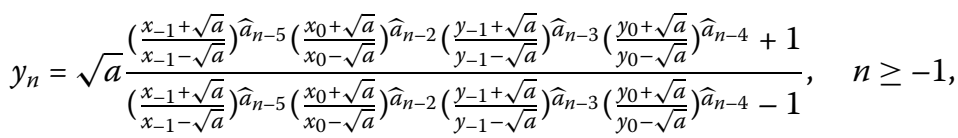

where the sequence $a_{n}$ is given by (164), while the sequence $\widehat{a}_{n}:=a_{n}+a_{n-1}$ is given by (166).

\subsection{Solution to system (83)}

By using the first equation in (83) into the second one, we get

$$
\zeta_{n}=\zeta_{n-1}^{2} \zeta_{n-2}^{-1} \zeta_{n-4}, \quad n \geq 3
$$

Let

$$
a_{0}=2, \quad b_{0}=-1, \quad c_{0}=0, \quad d_{0}=1 .
$$

Then equation (168) can be written as follows:

$$
\zeta_{n}=\zeta_{n-1}^{a_{0}} \zeta_{n-2}^{b_{0}} \zeta_{n-3}^{c_{0}} \zeta_{n-4}^{d_{0}}, \quad n \geq 3
$$

Similar to the previous case it is obtained that (152) and (153) hold for every $k, n \in \mathbb{N}$ such that $1 \leq k \leq n-3$. From (153) and (169), we have

$$
a_{k}=2 a_{k-1}+b_{k-1}, \quad b_{k}=-a_{k-1}+c_{k-1}, \quad c_{k}=d_{k-1}, \quad d_{k}=a_{k-1} .
$$

If in (152) we take $k=n-3$ and use (171), we get

$$
\begin{aligned}
\zeta_{n} & =\zeta_{2}^{a_{n-3}} \zeta_{1}^{b_{n-3}} \zeta_{0}^{c_{n-3}} \zeta_{-1}^{d_{n-3}} \\
& =\left(\zeta_{0} \eta_{-1} \eta_{0}\right)^{a_{n-3}}\left(\zeta_{0} \eta_{-1}\right)^{b_{n-3}} \zeta_{0}^{c_{n-3}} \zeta_{-1}^{d_{n-3}} \\
& =\zeta_{-1}^{d_{n-3}} \zeta_{0}^{a_{n-3}+b_{n-3}+c_{n-3}} \eta_{-1}^{a_{n-3}+b_{n-3}} \eta_{0}^{a_{n-3}} \\
& =\zeta_{-1}^{a_{n-4}} \zeta_{0}^{a_{n-1}-a_{n-2}} \eta_{-1}^{a_{n-2}-a_{n-3}} \eta_{0}^{a_{n-3}}
\end{aligned}
$$

for $n \geq 2$.

Using (172) into the relation $\eta_{n}=\zeta_{n+2} / \zeta_{n+1}$, we obtain

$$
\eta_{n}=\zeta_{-1}^{a_{n-2}-a_{n-3}} \zeta_{0}^{a_{n+1}-2 a_{n}+a_{n-1}} \eta_{-1}^{a_{n}-2 a_{n-1}+a_{n-2}} \eta_{0}^{a_{n-1}-a_{n-2}}
$$

From (153) and (169) we see that the sequences $\left(a_{k}\right)_{k \in \mathbb{N}_{0}},\left(b_{k}\right)_{k \in \mathbb{N}_{0}},\left(c_{k}\right)_{k \in \mathbb{N}_{0}}$, and $\left(d_{k}\right)_{k \in \mathbb{N}_{0}}$ are solutions to the following difference equation:

$$
w_{k}-2 w_{k-1}+w_{k-2}-w_{k-4}=0, \quad k \geq 4
$$


The roots of the characteristic polynomial

$$
P_{4}(t)=t^{4}-2 t^{3}+t^{2}-1=\left(t^{2}-t-1\right)\left(t^{2}-t+1\right)
$$

associated with the linear difference equation (174) are

$$
t_{1}=\frac{1+i \sqrt{3}}{2}, \quad t_{2}=\frac{1-i \sqrt{3}}{2}, \quad t_{3}=\lambda_{1}, \quad t_{4}=\lambda_{2} \text {. }
$$

The general solution to equation (174) has the following form:

$$
w_{k}=\alpha_{1} t_{1}^{k}+\alpha_{2} t_{2}^{k}+\alpha_{3} \lambda_{1}^{k}+\alpha_{4} \lambda_{2}^{k}, \quad k \in \mathbb{N}_{0}
$$

From (153) and (176) with $k=0,-1,-2,-3$, by some simple calculations, it follows that the equalities in (162) hold.

From (162), (176), and Lemma 1, it follows that

$$
a_{n}=\frac{t_{1}^{n+4}}{P_{4}^{\prime}\left(t_{1}\right)}+\frac{t_{2}^{n+4}}{P_{4}^{\prime}\left(t_{2}\right)}+\frac{\lambda_{1}^{n+4}}{P_{4}^{\prime}\left(\lambda_{1}\right)}+\frac{\lambda_{2}^{n+4}}{P_{4}^{\prime}\left(\lambda_{2}\right)}, \quad n \geq-4 .
$$

From this and since

$$
\begin{aligned}
& P_{4}^{\prime}\left(t_{1}\right)=\left(t_{1}-t_{2}\right)\left(t_{1}-\lambda_{1}\right)\left(t_{1}-\lambda_{2}\right)=-2 \sqrt{3} i, \\
& P_{4}^{\prime}\left(t_{2}\right)=\left(t_{2}-t_{1}\right)\left(t_{2}-\lambda_{1}\right)\left(t_{2}-\lambda_{2}\right)=2 \sqrt{3} i \\
& P_{4}^{\prime}\left(\lambda_{1}\right)=\left(\lambda_{1}-t_{1}\right)\left(\lambda_{1}-t_{2}\right)\left(\lambda_{1}-\lambda_{2}\right)=2\left(\lambda_{1}-\lambda_{2}\right)=2 \sqrt{5}, \\
& P_{4}^{\prime}\left(\lambda_{2}\right)=\left(\lambda_{2}-t_{1}\right)\left(\lambda_{2}-t_{2}\right)\left(\lambda_{2}-\lambda_{1}\right)=2\left(\lambda_{2}-\lambda_{1}\right)=-2 \sqrt{5},
\end{aligned}
$$

and after some calculation, we have

$$
\begin{aligned}
a_{n} & =\frac{i\left(t_{1}^{n+4}-t_{2}^{n+4}\right)}{2 \sqrt{3}}+\frac{\lambda_{1}^{n+4}-\lambda_{2}^{n+4}}{2\left(\lambda_{1}-\lambda_{2}\right)} \\
& =-\frac{1}{\sqrt{3}} \sin \frac{(n-2) \pi}{3}+\frac{f_{n+4}}{2}, \quad n \geq-4 .
\end{aligned}
$$

From (178), we have

$$
\begin{aligned}
a_{n}-a_{n-1} & =-\frac{1}{\sqrt{3}} \sin \frac{(n-2) \pi}{3}+\frac{f_{n+4}}{2}+\frac{1}{\sqrt{3}} \sin \frac{(n-3) \pi}{3}-\frac{f_{n+3}}{2} \\
& =-\frac{1}{\sqrt{3}} \cos \frac{(2 n-5) \pi}{6}+\frac{f_{n+2}}{2}
\end{aligned}
$$

and

$$
\begin{aligned}
a_{n}-2 a_{n-1}+a_{n-2} & =-\frac{1}{\sqrt{3}} \cos \frac{(2 n-5) \pi}{6}+\frac{f_{n+2}}{2}+\frac{1}{\sqrt{3}} \cos \frac{(2 n-7) \pi}{6}-\frac{f_{n+1}}{2} \\
& =-\frac{1}{\sqrt{3}} \sin \frac{n \pi}{3}+\frac{f_{n}}{2}
\end{aligned}
$$


Using (178) and (179) into (172) and (173), we obtain

$$
\zeta_{n}=\zeta_{-1}^{-\frac{1}{\sqrt{3}} \sin \frac{n \pi}{3}+\frac{f_{n}}{2}} \zeta_{0}^{-\frac{1}{\sqrt{3}} \cos \frac{(2 n-7) \pi}{6}+\frac{f_{n+1}}{2}} \eta_{-1}^{-\frac{1}{\sqrt{3}} \cos \frac{(2 n-9) \pi}{6}+\frac{f_{n}}{2}} \eta_{0}^{-\frac{1}{\sqrt{3}} \sin \frac{(n-5) \pi}{3}+\frac{f_{n+1}}{2}},
$$

and

$$
\begin{aligned}
\eta_{n}= & \zeta_{-1}^{-\frac{1}{\sqrt{3}} \cos \frac{(2 n-9) \pi}{6}+\frac{f_{n}}{2}} \zeta_{0}^{-\frac{1}{\sqrt{3}} \sin \frac{(n+1) \pi}{3}+\frac{f_{n+1}}{2}} \\
& \times \eta_{-1}^{-\frac{1}{\sqrt{3}} \sin \frac{n \pi}{3}+\frac{f_{n}}{2}} \eta_{0}^{-\frac{1}{\sqrt{3}} \cos \frac{(2 n-7) \pi}{6}+\frac{f_{n+1}}{2}},
\end{aligned}
$$

which are closed-form formulas for solutions to system (83).

Corollary 10 Assume that $k=0, l=1$, and $a \in \mathbb{C} \backslash\{0\}$. Then the general solution to system (49) is given by

$$
\begin{aligned}
& x_{n}=\sqrt{a} \frac{\left.\left(\frac{x_{-1}+\sqrt{a}}{x_{-1}-\sqrt{a}}\right)^{a_{n-4}}\left(\frac{x_{0}+\sqrt{a}}{x_{0}-\sqrt{a}}\right)^{\Delta a_{n-2}\left(\frac{y_{-1}+\sqrt{a}}{y_{-1}-\sqrt{a}}\right)}\right)^{\Delta a_{n-3}\left(\frac{y_{0}+\sqrt{a}}{y_{0}-\sqrt{a}}\right) a_{n-3}+1}}{\left(\frac{x_{-1}+\sqrt{a}}{x_{-1}-\sqrt{a}}\right) a_{n-4}\left(\frac{x_{0}+\sqrt{a}}{x_{0}-\sqrt{a}}\right) \Delta a_{n-2}\left(\frac{y_{-1}+\sqrt{a}}{y_{-1}-\sqrt{a}}\right) \Delta a_{n-3}\left(\frac{y_{0}+\sqrt{a}}{y_{0}-\sqrt{a}}\right) a_{n-3}-1}, \quad n \geq-1,
\end{aligned}
$$

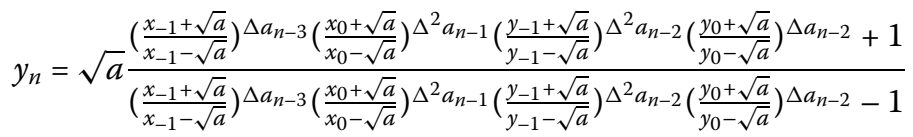

for $n \geq-1$, where the sequence $a_{n}$ is given by (178), $\Delta a_{n-1}$ by (179), and $\Delta^{2} a_{n-2}$ by (180).

\subsection{Solution to system (84)}

From the equations in (84) we see that $\zeta_{n}=\eta_{n}, n \in \mathbb{N}$. Hence, $\zeta_{n+1}=\zeta_{n} \zeta_{n-1}, n \geq 2$.

From this, (90) where $\zeta_{-1}$ and $\zeta_{0}$ are replaced by $\zeta_{1}$ and $\zeta_{2}$ respectively and (8), we have that

$$
\begin{aligned}
\zeta_{n} & =\zeta_{2}^{f_{n-1}} \zeta_{1}^{f_{n-2}} \\
& =\left(\zeta_{0} \eta_{-1} \eta_{0}\right)^{f_{n-1}}\left(\zeta_{0} \eta_{-1}\right)^{f_{n-2}} \\
& =\zeta_{0}^{f_{n}} \eta_{-1}^{f_{n}} \eta_{0}^{f_{n-1}}, \quad n \in \mathbb{N},
\end{aligned}
$$

and consequently

$$
\eta_{n}=\zeta_{0}^{f_{n}} \eta_{-1}^{f_{n}} \eta_{0}^{f_{n-1}}, \quad n \in \mathbb{N} .
$$

It is easy to see that formula (184) holds also for $n=0$.

Formulas (183) and (184) are the closed-form formulas for general solution to system (84).

Corollary 11 Assume that $k=0, l=1$, and $a \in \mathbb{C} \backslash\{0\}$. Then the general solution to system (50) is given by

$$
\begin{aligned}
& x_{n}=\sqrt{a} \frac{\left(\frac{x_{0}+\sqrt{a}}{x_{0}-\sqrt{a}}\right)^{f_{n}}\left(\frac{y_{0}+\sqrt{a}}{y_{0}-\sqrt{a}}\right) f_{n-1}\left(\frac{y_{-1}+\sqrt{a}}{y_{1}-\sqrt{a}}\right)^{f_{n}}+1}{\left(\frac{x_{0}+\sqrt{a}}{x_{0}-\sqrt{a}}\right) f_{n}\left(\frac{y_{0}+\sqrt{a}}{y_{0}-\sqrt{a}}\right) f_{n-1}\left(\frac{y_{-1}+\sqrt{a}}{y_{1}-\sqrt{a}}\right) f_{n}-1}, \quad n \in \mathbb{N}, \\
& y_{n}=\sqrt{a} \frac{\left(\frac{x_{0}+\sqrt{a}}{x_{0}-\sqrt{a}}\right)^{f_{n}}\left(\frac{y_{0}+\sqrt{a}}{y_{0}-\sqrt{a}}\right)^{f_{n-1}\left(\frac{y_{-1}+\sqrt{a}}{y_{1}-\sqrt{a}}\right)^{f_{n}}+1}}{\left(\frac{x_{0}+\sqrt{a}}{x_{0}-\sqrt{a}}\right)_{n}\left(\frac{y_{0}+\sqrt{a}}{y_{0}-\sqrt{a}}\right) f_{n-1}\left(\frac{y-1+\sqrt{a}}{y_{1}-\sqrt{a}}\right) f_{n}-1}, \quad n \in \mathbb{N}_{0} .
\end{aligned}
$$




\subsection{Solution to system (85)}

This system is obtained from system (75) by interchanging letters $\zeta$ and $\eta$. Hence, its solution is given by

$$
\zeta_{n}=\zeta_{0} \eta_{0}^{f_{n+1}-1} \eta_{-1}^{f_{n}}, \quad n \in \mathbb{N}_{0}
$$

and

$$
\eta_{n}=\eta_{0}^{f_{n+1}} \eta_{-1}^{f_{n}}, \quad n \geq-1
$$

Formulas (185) and (186) are the closed-form formulas for general solution to system (85).

Corollary 12 Assume that $k=0, l=1$, and $a \in \mathbb{C} \backslash\{0\}$. Then the general solution to system (51) is given by

$$
\begin{aligned}
& x_{n}=\sqrt{a} \frac{\left(\frac{x_{0}+\sqrt{a}}{x_{0}-\sqrt{a}}\right)\left(\frac{y_{0}+\sqrt{a}}{y_{0}-\sqrt{a}}\right)^{f_{n+1}-1}\left(\frac{y_{-1}+\sqrt{a}}{y_{-1}-\sqrt{a}}\right)^{f_{n}}+1}{\left(\frac{x_{0}+\sqrt{a}}{x_{0}-\sqrt{a}}\right)\left(\frac{y_{0}+\sqrt{a}}{y_{0}-\sqrt{a}}\right)^{f_{n+1}-1}\left(\frac{y_{-1}+\sqrt{a}}{y_{-1}-\sqrt{a}}\right)^{f_{n}}-1}, \quad n \in \mathbb{N}_{0}, \\
& y_{n}=\sqrt{a} \frac{\left(\frac{y_{0}+\sqrt{a}}{y_{0}-\sqrt{a}}\right) f_{n+1}^{f_{n}\left(\frac{y_{-1}+\sqrt{a}}{y_{-1}-\sqrt{a}}\right)^{f_{n}}+1}}{\left(\frac{y_{0}+\sqrt{a}}{y_{0}-\sqrt{a}}\right) f_{n+1}\left(\frac{y_{-1}+\sqrt{a}}{y_{-1}-\sqrt{a}}\right)^{f_{n}}-1}, \quad n \geq-1 .
\end{aligned}
$$

\subsection{Solution to system (86)}

By using the second equation in (86) into the first one, we get

$$
\zeta_{n}=\zeta_{n-2} \zeta_{n-3}^{2} \zeta_{n-4}, \quad n \geq 3
$$

Let

$$
b_{0}=1, \quad c_{0}=2, \quad d_{0}=1, \quad e_{0}=0 .
$$

Then equation (188) can be written as follows:

$$
\zeta_{n}=\zeta_{n-2}^{b_{0}} \zeta_{n-3}^{c_{0}} \zeta_{n-4}^{d_{0}} \zeta_{n-5}^{e_{0}}, \quad n \geq 3
$$

Similar to the case of equation (78) it is obtained that

$$
\zeta_{n}=\zeta_{n-k-2}^{b_{k}} \zeta_{n-k-3}^{c_{k}} \zeta_{n-k-4}^{d_{k}} \zeta_{n-k-5}^{e_{k}}
$$

for $n \geq k+4$, and that

$$
b_{k}=c_{k-1}, \quad c_{k}=b_{0} b_{k-1}+d_{k-1}, \quad d_{k}=c_{0} b_{k-1}+e_{k-1}, \quad e_{k}=d_{0} b_{k-1}
$$

for $k \in \mathbb{N}$.

Using (188) in (191), we have

$$
b_{k}=c_{k-1}, \quad c_{k}=b_{k-1}+d_{k-1}, \quad d_{k}=2 b_{k-1}+e_{k-1}, \quad e_{k}=b_{k-1} .
$$


If in (190) we take $k=n-4$ and calculate $\zeta_{1}$ and $\zeta_{2}$, we get

$$
\begin{aligned}
\zeta_{n} & =\zeta_{2}^{b_{n-4}} \zeta_{1}^{c_{n-4}} \zeta_{0}^{d_{n-4}} \zeta_{-1}^{e_{n-4}} \\
& =\left(\zeta_{0} \zeta_{-1} \eta_{0}\right)^{b_{n-4}}\left(\eta_{0} \eta_{-1}\right)^{c_{n-4}} \zeta_{0}^{d_{n-4}} \zeta_{-1}^{e_{n-4}} \\
& =\zeta_{-1}^{b_{n-4}+e_{n-4}} \zeta_{0}^{b_{n-4}+d_{n-4}} \eta_{-1}^{c_{n-4}} \eta_{0}^{b_{n-4}+c_{n-4}} \\
& =\zeta_{-1}^{b_{n-4}+b_{n-5}} \zeta_{0}^{b_{n-2}} \eta_{-1}^{b_{n-3}} \eta_{0}^{b_{n-3}+b_{n-4}}
\end{aligned}
$$

for $n \geq 4$.

Using (193) into the second equation in (86) and employing (192), we obtain

$$
\eta_{n}=\zeta_{n-1} \zeta_{n-2}=\zeta_{-1}^{b_{n-3}} \zeta_{0}^{b_{n-3}+b_{n-4}} \eta_{-1}^{b_{n-4}+b_{n-5}} \eta_{0}^{b_{n-2}}
$$

From (192) we see that the sequences $\left(b_{k}\right)_{k \in \mathbb{N}_{0}},\left(c_{k}\right)_{k \in \mathbb{N}_{0}},\left(d_{k}\right)_{k \in \mathbb{N}_{0}}$, and $\left(e_{k}\right)_{k \in \mathbb{N}_{0}}$ are solutions to the following difference equation:

$$
w_{k}-w_{k-2}-2 w_{k-3}-w_{k-4}=0, \quad k \geq 4 .
$$

From (192) for $k=0,-1,-2,-3,-4$, it is obtained $b_{-1}=0, c_{-1}=1, d_{-1}=2, e_{-1}=1$,

$$
\begin{array}{llll}
b_{-2}=1, & c_{-2}=0, & d_{-2}=0, & e_{-2}=0, \\
b_{-3}=0, & c_{-3}=1, & d_{-3}=0, & e_{-3}=0, \\
b_{-4}=0, & c_{-4}=0, & d_{-4}=1, & e_{-4}=0, \\
b_{-5}=0, & c_{-5}=0, & d_{-5}=0, & e_{-5}=1 .
\end{array}
$$

The roots of the characteristic polynomial

$$
P_{4}(t)=t^{4}-t^{2}-2 t-1=\left(t^{2}-t-1\right)\left(t^{2}+t+1\right)
$$

associated with equation (195) are

$$
t_{1}=\frac{-1+i \sqrt{3}}{2}, \quad t_{2}=\frac{-1-i \sqrt{3}}{2}, \quad t_{3}=\lambda_{1}, \quad t_{4}=\lambda_{2} .
$$

The general solution to equation (195) is

$$
w_{k}=\alpha_{1} t_{1}^{k}+\alpha_{2} t_{2}^{k}+\alpha_{3} \lambda_{1}^{k}+\alpha_{4} \lambda_{2}^{k}, \quad k \in \mathbb{N}_{0} .
$$

From (196), (198), and Lemma 1, it follows that

$$
b_{n}=\frac{t_{1}^{n+5}}{P_{4}^{\prime}\left(t_{1}\right)}+\frac{t_{2}^{n+5}}{P_{4}^{\prime}\left(t_{2}\right)}+\frac{\lambda_{1}^{n+5}}{P_{4}^{\prime}\left(\lambda_{1}\right)}+\frac{\lambda_{2}^{n+5}}{P_{4}^{\prime}\left(\lambda_{2}\right)}, \quad n \geq-5 .
$$


From this and since

$$
\begin{aligned}
& P_{4}^{\prime}\left(t_{1}\right)=\left(t_{1}-t_{2}\right)\left(t_{1}-\lambda_{1}\right)\left(t_{1}-\lambda_{2}\right)=3-i \sqrt{3}=2 \sqrt{3} i t_{2}, \\
& P_{4}^{\prime}\left(t_{2}\right)=\left(t_{2}-t_{1}\right)\left(t_{2}-\lambda_{1}\right)\left(t_{2}-\lambda_{2}\right)=3+i \sqrt{3}=-2 \sqrt{3} i t_{1}, \\
& P_{4}^{\prime}\left(\lambda_{1}\right)=\left(\lambda_{1}-t_{1}\right)\left(\lambda_{1}-t_{2}\right)\left(\lambda_{1}-\lambda_{2}\right)=5+3 \sqrt{5}=2 \sqrt{5} \lambda_{1}^{2}, \\
& P_{4}^{\prime}\left(\lambda_{2}\right)=\left(\lambda_{2}-t_{1}\right)\left(\lambda_{2}-t_{2}\right)\left(\lambda_{2}-\lambda_{1}\right)=5-3 \sqrt{5}=-2 \sqrt{5} \lambda_{2}^{2},
\end{aligned}
$$

and after some calculation, we have

$$
\begin{aligned}
b_{n} & =\frac{i\left(t_{2}^{n+6}-t_{1}^{n+6}\right)}{2 \sqrt{3}}+\frac{\lambda_{1}^{n+3}-\lambda_{2}^{n+3}}{2\left(\lambda_{1}-\lambda_{2}\right)} \\
& =-\frac{1}{\sqrt{3}} \sin \frac{4 n \pi}{3}+\frac{f_{n+3}}{2}, \quad n \geq-4 .
\end{aligned}
$$

From (200), we have

$$
\begin{aligned}
\widehat{b}_{n} & :=b_{n}+b_{n-1}=-\frac{1}{\sqrt{3}} \sin \frac{4 n \pi}{3}+\frac{f_{n+3}}{2}-\frac{1}{\sqrt{3}} \sin \frac{4(n-1) \pi}{3}+\frac{f_{n+2}}{2} \\
& =\frac{1}{\sqrt{3}} \sin \frac{2(2 n-1) \pi}{3}+\frac{f_{n+4}}{2}
\end{aligned}
$$

for $n \geq-3$.

Using (200) and (201) into (193) and (194), we obtain

$$
\begin{aligned}
\zeta_{n}= & \zeta_{-1}^{b_{n-4}+b_{n-5}} \zeta_{0}^{b_{n-2}} \eta_{-1}^{b_{n-3}} \eta_{0}^{b_{n-3}+b_{n-4}} \\
= & \zeta_{-1}^{\frac{1}{\sqrt{3}} \sin \frac{2(2 n-9) \pi}{3}+\frac{f_{n}}{2}} \zeta_{0}^{-\frac{1}{\sqrt{3}} \sin \frac{4(n-2) \pi}{3}+\frac{f_{n+1}}{2}} \\
& \times \eta_{-1}^{-\frac{1}{\sqrt{3}} \sin \frac{4(n-3) \pi}{3}+\frac{f_{n}}{2}} \eta_{0}^{\frac{1}{\sqrt{3}} \sin \frac{2(2 n-7) \pi}{3}+\frac{f_{n+1}}{2}},
\end{aligned}
$$

and

$$
\begin{aligned}
\eta_{n}= & \zeta_{-1}^{b_{n-3}} \zeta_{0}^{b_{n-3}+b_{n-4}} \eta_{-1}^{b_{n-4}+b_{n-5}} \eta_{0}^{b_{n-2}} \\
= & \zeta_{-1}^{-\frac{1}{\sqrt{3}} \sin \frac{4(n-3) \pi}{3}+\frac{f_{n}}{2}} \zeta_{0}^{\frac{1}{\sqrt{3}} \sin \frac{2(2 n-7) \pi}{3}+\frac{f_{n+1}}{2}} \\
& \times \eta_{-1}^{\frac{1}{\sqrt{3}} \sin \frac{2(2 n-9) \pi}{3}+\frac{f_{n}}{2}} \eta_{0}^{-\frac{1}{\sqrt{3}} \sin \frac{4(n-2) \pi}{3}+\frac{f_{n+1}}{2}},
\end{aligned}
$$

which are closed-form formulas for solutions to system (86).

Corollary 13 Assume that $k=0, l=1$, and $a \in \mathbb{C} \backslash\{0\}$. Then the general solution to system (52) is given by

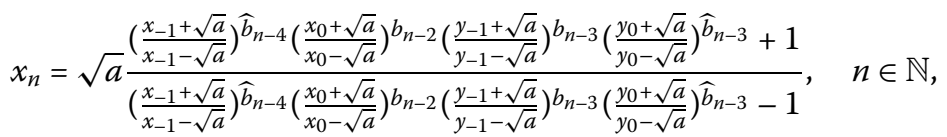

$$
\begin{aligned}
& y_{n}=\sqrt{a} \frac{\left(\frac{x_{-1}+\sqrt{a}}{x_{-1}-\sqrt{a}}\right)^{b_{n-3}}\left(\frac{x_{0}+\sqrt{a}}{x_{0}-\sqrt{a}}\right)^{b_{n-3}}\left(\frac{y_{-1}+\sqrt{a}}{y_{-1}-\sqrt{a}}\right)^{x_{n-4}}\left(\frac{y_{0}+\sqrt{a}}{y_{0}-\sqrt{a}}\right)^{b_{n-2}}+1}{\left(\frac{x_{-1}+\sqrt{a}}{x_{-1}-\sqrt{a}}\right)^{b_{n-3}}\left(\frac{x_{0}+\sqrt{a}}{x_{0}-\sqrt{a}}\right)^{b_{n-3}}\left(\frac{y_{-1}+\sqrt{a}}{y_{-1}-\sqrt{a}}\right)^{b_{n-4}}\left(\frac{y_{0}+\sqrt{a}}{y_{0}-\sqrt{a}}\right)^{b_{n-2}-1}}, \quad n \in \mathbb{N},
\end{aligned}
$$

where the sequence $b_{n}$ is given by (200), while $\widehat{b}_{n}$ is given by (201). 


\subsection{Solution to system (87)}

This system is obtained from system (82) by interchanging letters $\zeta$ and $\eta$. Hence, its solution is given by

$$
\begin{aligned}
\zeta_{n}= & \eta_{-1}^{\frac{\operatorname{Re}\left((3+i) i^{n-1}\right)+\lambda_{1}^{n-1}+\lambda_{2}^{n-1}}{5}} \eta_{0}^{\frac{\operatorname{Re}\left((3+i) i^{n-2}\right)+\lambda_{1}^{n+2}+\lambda_{2}^{n+2}}{5}} \\
& \times \zeta_{-1}^{\frac{\operatorname{Re}\left((3+i) i^{n-3}\right)+\lambda_{1}^{n+1}+\lambda_{2}^{n+1}}{5}} \zeta_{0}^{\frac{\operatorname{Re}\left((3+i) i^{n}\right)+\lambda_{1}^{n}+\lambda_{2}^{n}}{5}}
\end{aligned}
$$

and

$$
\begin{aligned}
\eta_{n}= & \eta_{-1}^{\frac{\operatorname{Re}\left((1+2 i) i^{n}\right)+\lambda_{1}^{n-1}+\lambda_{2}^{n-1}}{5}} \eta_{0}^{\frac{\operatorname{Re}\left((1+2 i) i^{n-1}\right)+\lambda_{1}^{n+2}+\lambda_{2}^{n+2}}{5}} \\
& \times \zeta_{-1}^{\frac{\operatorname{Re}\left((1+2 i) i^{n-2}\right)+\lambda_{1}^{n+1}+\lambda_{2}^{n+1}}{5}} \zeta_{0}^{\frac{\operatorname{Re}\left((1+2 i) i^{n-3}\right)+\lambda_{1}^{n}+\lambda_{2}^{n}}{5}}
\end{aligned}
$$

for $n \geq 2$.

Formulas (204) and (205) present the closed-form formulas for general solution to system (87).

Corollary 14 Assume that $k=0, l=1$, and $a \in \mathbb{C} \backslash\{0\}$. Then the general solution to system (53) is given by

$$
\begin{aligned}
& x_{n}=\sqrt{a} \frac{\left(\frac{y_{-1}+\sqrt{a}}{y_{-1}-\sqrt{a}}\right)^{\widehat{a}_{n-5}}\left(\frac{y_{0}+\sqrt{a}}{y_{0}-\sqrt{a}}\right)^{\widehat{a}_{n-2}}\left(\frac{x_{-1}+\sqrt{a}}{x_{-1}-\sqrt{a}}\right) \widehat{a}_{n-3}\left(\frac{x_{0}+\sqrt{a}}{x_{0}-\sqrt{a}}\right)^{\widehat{a}_{n-4}}+1}{\left(\frac{y_{-1}+\sqrt{a}}{y_{-1}-\sqrt{a}}\right) \widehat{a}_{n-5}\left(\frac{y_{0}+\sqrt{a}}{y_{0}-\sqrt{a}}\right) \widehat{a}_{n-2}\left(\frac{x_{-1}+\sqrt{a}}{x_{-1}-\sqrt{a}}\right) \widehat{a}_{n-3}\left(\frac{x_{0}+\sqrt{a}}{x_{0}-\sqrt{a}}\right)^{\widehat{a}_{n-4}-1}}, \quad n \geq-1,
\end{aligned}
$$

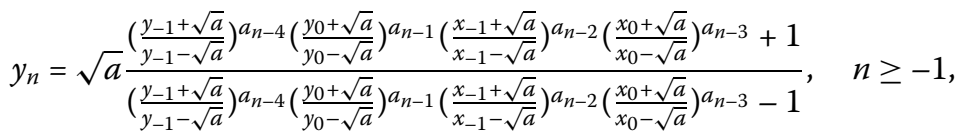

where the sequence $a_{n}$ is given by (164), while the sequence $\widehat{a}_{n}:=a_{n}+a_{n-1}$ is given by (166).

\subsection{Solution to system (88)}

This system is obtained from system (78) by interchanging letters $\zeta$ and $\eta$. Hence, its solution is given by

$$
\eta_{n}=\zeta_{0}^{(-1)^{n-1}+f_{n-1}} \eta_{0}^{(-1)^{n}+f_{n}} \eta_{-1}^{f_{n}}, \quad n \geq-1,
$$

and

$$
\zeta_{n}=\zeta_{0}^{f_{n-1}} \eta_{0}^{f_{n}} \eta_{-1}^{f_{n}}
$$

for $n \in \mathbb{N}_{0}$.

Corollary 15 Assume that $k=0, l=1$, and $a \in \mathbb{C} \backslash\{0\}$. Then the general solution to system (54) is given by

$$
\begin{aligned}
& x_{n}=\sqrt{a} \frac{\left(\frac{x_{0}+\sqrt{a}}{x_{0}-\sqrt{a}}\right)^{f_{n-1}}\left(\frac{y_{0}+\sqrt{a}}{y_{0}-\sqrt{a}}\right)^{f_{n}}\left(\frac{y_{-1}+\sqrt{a}}{y_{-1}-\sqrt{a}}\right)^{f_{n}}+1}{\left(\frac{x_{0}+\sqrt{a}}{x_{0}-\sqrt{a}}\right) f_{n-1}\left(\frac{y_{0}+\sqrt{a}}{y_{0}-\sqrt{a}}\right) f_{n}\left(\frac{y_{-1}+\sqrt{a}}{y_{-1}-\sqrt{a}}\right)^{f_{n}}-1}, \quad n \in \mathbb{N}_{0},
\end{aligned}
$$

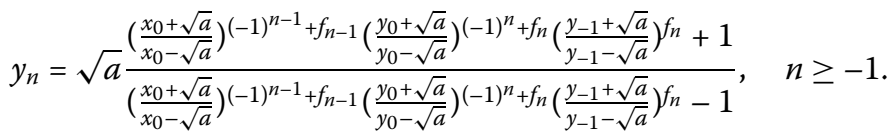




\subsection{Solution to system (89)}

This system of difference equations is obtained from system (74) by interchanging letters $\zeta$ and $\eta$ only. Hence, its solution is given by

$$
\eta_{n}=\eta_{0}^{f_{n+1}} \eta_{-1}^{f_{n}}, \quad n \geq-1
$$

and consequently

$$
\zeta_{n}=\eta_{0}^{f_{n+1}} \eta_{-1}^{f_{n}}
$$

for $n \in \mathbb{N}$.

Formulas (208) and (209) are the closed-form formulas for general solution to system (89).

Corollary 16 Assume that $k=0, l=1$, and $a \in \mathbb{C} \backslash\{0\}$. Then the general solution to system (55) is given by

$$
\begin{aligned}
& x_{n}=\sqrt{a} \frac{\left(\frac{y_{0}+\sqrt{a}}{y_{0}-\sqrt{a}}\right)^{f_{n+1}}\left(\frac{y_{-1}+\sqrt{a}}{y_{-1}-\sqrt{a}}\right)^{f_{n}}+1}{\left(\frac{y_{0}+\sqrt{a}}{y_{0}-\sqrt{a}}\right) f_{n+1}\left(\frac{y_{-1}+\sqrt{a}}{y_{-1}-\sqrt{a}}\right)^{f_{n}}-1}, \quad n \in \mathbb{N}, \\
& y_{n}=\sqrt{a} \frac{\left(\frac{y_{0}+\sqrt{a}}{y_{0}-\sqrt{a}}\right)^{f_{n+1}\left(\frac{y_{-1}+\sqrt{a}}{y_{-1}-\sqrt{a}}\right)^{f_{n}}+1}}{\left(\frac{y_{0}+\sqrt{a}}{y_{0}-\sqrt{a}}\right) f_{n+1}\left(\frac{y_{-1}+\sqrt{a}}{y_{-1}-\sqrt{a}}\right)^{f_{n}}-1}, \quad n \geq-1 .
\end{aligned}
$$

Remark 2 As we have seen in Sect. 2, by using the changes of variables (13) the systems in (12) are transformed to some two-dimensional linear systems of difference equations with constant coefficients, which are further transformed to some linear difference equations with constant coefficients, which are solvable. Hence, the systems in (11) when $a=0$ are easier to solve than the ones in the case $a \neq 0$. Bearing in mind the fact that the case $k=$ $0, l=1$ was essentially treated in [62] and [63] and that the main aim of the paper is to present the above method for solving the systems for the case $a \neq 0$, we left the case to the interested reader as an exercise.

Remark 3 The case $k=1, l=2$, is treated in [70]. The case $k=0, l=2$, can be also treated by using the method presented here.

Acknowledgements

The study in this paper is a part of the investigations under the projects III 41025 and III 44006 by the Serbian Ministry of Education and Science.

Funding

Not applicable.

Availability of data and materials

Not applicable.

Competing interests

The author declares that he has no competing interests. 


\section{Author details}

'Mathematical Institute of the Serbian Academy of Sciences, Beograd, Serbia. ${ }^{2}$ Department of Medical Research, China Medical University Hospital, China Medical University, Taichung, Taiwan, Republic of China. ${ }^{3}$ Department of Computer Science and Information Engineering, Asia University, Taichung, Taiwan, Republic of China.

\section{Publisher's Note}

Springer Nature remains neutral with regard to jurisdictional claims in published maps and institutional affiliations.

Received: 7 June 2019 Accepted: 8 July 2019 Published online: 18 July 2019

\section{References}

1. de Moivre, A.: De Fractionibus algebraicis radicalitate immunibus ad fractiones simpliciores reducendis, deque summandis terminis quarumdam serierum aequali intervallo a se distantibus. Philos. Trans. 32, 162-178 (1722) (in Latin)

2. Laplace, P.S.: Recherches sur l'intégration des équations différentielles aux différences finies et sur leur usage dans la théorie des hasards. Mémoires de l'Académie Royale des Sciences de Paris 1773 VII (1776); Laplace OEuvres VIII 69-197 (1891) (in French)

3. de Moivre, A.: Miscellanea analytica de seriebus et quadraturis. Londini (1730) (in Latin)

4. Euler, L.: Introductio in Analysin Infinitorum, Tomus Primus. Lausannae (1748)

5. Lagrange, J.-L.: OEuvres, t. II. Gauthier-Villars, Paris (1868) (in French)

6. Boole, G.: A Treatsie on the Calculus of Finite Differences, 3rd edn. Macmillan \& Co., London (1880)

7. Markov, A.A.: Ischislenie Konechnykh Raznostey, 2nd edn. Matezis, Odessa (1910) (in Russian)

8. Fort, T.: Finite Differences and Difference Equations in the Real Domain. Oxford University Press, London (1948)

9. Jordan, C.: Calculus of Finite Differences. Chelsea, New York (1956)

10. Milne-Thomson, L.M.: The Calculus of Finite Differences. Macmillan \& Co., London (1933)

11. Mitrinović, D.S., Kečkić, J.D.: Methods for Calculating Finite Sums. Naučna Knjiga, Beograd (1984) (in Serbian)

12. Nörlund, N.E.: Vorlesungen über Differenzenrechnung. Springer, Berlin (1924) (in German)

13. Stević, S.: Representation of solutions of bilinear difference equations in terms of generalized Fibonacci sequences. Electron. J. Qual. Theory Differ. Equ. 2014, Article ID 67 (2014)

14. Stević, S.: Representations of solutions to linear and bilinear difference equations and systems of bilinear difference equations. Adv. Differ. Equ. 2018, Article ID 474 (2018)

15. Stević, S., Diblik, J., Iričanin, B., Šmarda, Z.: On some solvable difference equations and systems of difference equations. Abstr. Appl. Anal. 2012, Article ID 541761 (2012)

16. Stević, S., Iričanin, B., Kosmala, W., Šmarda, Z.: Note on the bilinear difference equation with a delay. Math. Methods Appl. Sci. 41, 9349-9360 (2018)

17. Stević, S., Iričanin, B., Kosmala, W., Šmarda, Z.: Representation of solutions of a solvable nonlinear difference equation of second order. Electron. J. Qual. Theory Differ. Equ. 2018, Article ID 95 (2018)

18. Stević, S., Iričanin, B., Šmarda, Z.: On a symmetric bilinear system of difference equations. Appl. Math. Lett. 89, 15-21 (2019)

19. Papaschinopoulos, G., Stefanidou, G.: Asymptotic behavior of the solutions of a class of rational difference equations. Int. J. Difference Equ. 5(2), 233-249 (2010)

20. Berg, L., Stević, S.: On some systems of difference equations. Appl. Math. Comput. 218, 1713-1718 (2011)

21. Stević, S.: On the difference equation $x_{n}=x_{n-2} /\left(b_{n}+c_{n} x_{n-1} x_{n-2}\right)$. Appl. Math. Comput. 218, 4507-4513 (2011)

22. Stević, S:: On the difference equation $x_{n}=x_{n-k} /\left(b+c x_{n-1} \cdots x_{n-k}\right)$. Appl. Math. Comput. 218, 6291-6296 (2012)

23. Stević, S.: On the system of difference equations $x_{n}=c_{n} y_{n-3} /\left(a_{n}+b_{n} y_{n-1} x_{n-2} y_{n-3}\right), y_{n}=\gamma_{n} x_{n-3} /\left(\alpha_{n}+\beta_{n} x_{n-1} y_{n-2} x_{n-3}\right)$. Appl. Math. Comput. 219, 4755-4764 (2013)

24. Stević, S., Diblik, J., Iričanin, B., Šmarda, Z.: On a third-order system of difference equations with variable coefficients Abstr. Appl. Anal. 2012, Article ID 508523 (2012)

25. Andruch-Sobilo, A., Migda, M.: Further properties of the rational recursive sequence $x_{n+1}=a x_{n-1} /\left(b+c x_{n} x_{n-1}\right)$. Opusc. Math. 26(3), 387-394 (2006)

26. Papaschinopoulos, G., Schinas, C.J.: On a system of two nonlinear difference equations. J. Math. Anal. Appl. 219(2) 415-426 (1998)

27. Papaschinopoulos, G., Schinas, C.J.: On the behavior of the solutions of a system of two nonlinear difference equations. Commun. Appl. Nonlinear Anal. 5(2), 47-59 (1998)

28. Papaschinopoulos, G., Schinas, C.J.: Invariants for systems of two nonlinear difference equations. Differ. Equ. Dyn. Syst. 7. 181-196 (1999)

29. Papaschinopoulos, G., Schinas, C.J.: Invariants and oscillation for systems of two nonlinear difference equations. Nonlinear Anal., Theory Methods Appl. 46, 967-978 (2001)

30. Papaschinopoulos, G., Schinas, C.J.: Oscillation and asymptotic stability of two systems of difference equations of rational form. J. Differ. Equ. Appl. 7, 601-617 (2001)

31. Papaschinopoulos, G., Schinas, C.J., Stefanidou, G.: On a k-order system of Lyness-type difference equations. Adv. Differ. Equ. 2007, Article ID 31272 (2007)

32. Stefanidou, G., Papaschinopoulos, G., Schinas, C.: On a system of max difference equations. Dyn. Contin. Discrete Impuls. Syst., Ser. A Math. Anal. 14(6), 885-903 (2007)

33. Schinas, C.: Invariants for difference equations and systems of difference equations of rational form. J. Math. Anal. Appl. 216, 164-179 (1997)

34. Schinas, C.: Invariants for some difference equations. J. Math. Anal. Appl. 212, 281-291 (1997)

35. Stević, S.: First-order product-type systems of difference equations solvable in closed form. Electron. J. Differ. Equ. 2015, Article ID 308 (2015)

36. Stević, S.: Product-type system of difference equations of second-order solvable in closed form. Electron. J. Qual. Theory Differ. Equ. 2015, Article ID 56 (2015) 
37. Stević, S.: Solvable product-type system of difference equations whose associated polynomial is of the fourth order. Electron. J. Qual. Theory Differ. Equ. 2017, Article ID 13 (2017)

38. Stević, S.: Solvability of a product-type system of difference equations with six parameters. Adv. Nonlinear Anal. 8(1), 29-51 (2019)

39. Stević, S., Iričanin, B., Šmarda, Z:: On a product-type system of difference equations of second order solvable in closed form. J. Inequal. Appl. 2015, Article ID 327 (2015)

40. Stević, S., Iričanin, B., Šmarda, Z.: Solvability of a close to symmetric system of difference equations. Electron. J. Differ. Equ. 2016, Article ID 159 (2016)

41. Stević, S., Iričanin, B., Šmarda, Z: Two-dimensional product-type system of difference equations solvable in closed form. Adv. Differ. Equ. 2016, Article ID 253 (2016)

42. Stević, S., Alghamdi, M.A., Alotaibi, A., Elsayed, E.M.: Solvable product-type system of difference equations of second order. Electron. J. Differ. Equ. 2015, Article ID 169 (2015)

43. Stević, S.: Solvability of a one-parameter class of nonlinear second-order difference equations by invariants. Adv. Differ. Equ. 2019, Article ID 151 (2019)

44. Berezansky, L., Braverman, E.: On impulsive Beverton-Holt difference equations and their applications. J. Differ. Equ. Appl. 10(9), 851-868 (2004)

45. Krechmar, V.A.: A Problem Book in Algebra. Mir, Moscow (1974)

46. Levy, H., Lessman, F.: Finite Difference Equations. Dover, New York (1992)

47. Mitrinović, D.S.: Matrices and Determinants. Naučna Knjiga, Beograd (1989) (in Serbian)

48. Mitrinović, D.S., Adamović, D.D.: Sequences and Series. Naučna Knjiga, Beograd (1980) (in Serbian)

49. Proskuryakov, I.V.: Problems in Linear Algebra. Nauka, Moscow (1984) (in Russian)

50. Riordan, J.: Combinatorial Identities. Wiley, New York (1968)

51. Iričanin, B., Stević, S.: Eventually constant solutions of a rational difference equation. Appl. Math. Comput. 215 854-856 (2009)

52. Stević, S.: On some periodic systems of max-type difference equations. Appl. Math. Comput. 218, 11483-11487 (2012)

53. Stević, S.: Bounded and periodic solutions to the linear first-order difference equation on the integer domain. Adv. Differ. Equ. 2017, Article ID 283 (2017)

54. Stević, S.: Bounded solutions to nonhomogeneous linear second-order difference equations. Symmetry 9, Article ID $227(2017)$

55. Stević, S.: Existence of a unique bounded solution to a linear second order difference equation and the linear first order difference equation. Adv. Differ. Equ. 2017, Article ID 169 (2017)

56. Kruse, N., Nesemann, T.: Global asymptotic stability in some discrete dynamical systems. J. Math. Anal. Appl. 235, 151-158 (1999)

57. Berg, L., Stević, S.: On the asymptotics of some difference equations. J. Differ. Equ. Appl. 18(5), 785-797 (2012)

58. Papaschinopoulos, G., Schinas, C.J.: Oscillation and asymptotic stability of two systems of difference equations of rational form. J. Differ. Equ. Appl. 7, 601-617 (2001)

59. Diblik, J., Iričanin, B., Stević, S., Šmarda, Z.: On some symmetric systems of difference equations. Abstr. Appl. Anal. 2013, Article ID 246723 (2013)

60. Rhouma, M.H.: The Fibonacci sequence modulo $\pi$, chaos and some rational recursive equations. J. Math. Anal. Appl. 310, 506-517 (2005)

61. Stević, S., Iričanin, B., Kosmala, W.: More on a hyperbolic-cotangent class of difference equations. Math. Methods Appl. Sci. 42, 2974-2992 (2019)

62. Stević, S., Tollu, D.T.: Solvability and semi-cycle analysis of a class of nonlinear systems of difference equations. Math. Methods Appl. Sci. 42, 3579-3615 (2019)

63. Stević, S., Tollu, D.T.: Solvability of eight classes of nonlinear systems of difference equations. Math. Methods Appl. Sci. 42, 4065-4112 (2019)

64. Stević, S.: On some solvable systems of difference equations. Appl. Math. Comput. 218, 5010-5018 (2012)

65. Adamović, D.: Solution to problem 194. Mat. Vesn. 23, 236-242 (1971)

66. Alfred, B.U.: An Introduction to Fibonacci Discovery. The Fibonacci Association (1965)

67. Vorobiev, N.N.: Fibonacci Numbers. Birkhäuser, Basel (2002) (Russian original 1950)

68. Abel, N.H.: Mémoire sur les équations algébriques, ou l'on démontre l'impossibilité de la résolution de l'équation générale du cinquième degré. In: Sylow, L., Lie, S. (eds.) Oeuvres Complètes de Niels Henrik Abel, Vol. I, 2nd edn. pp. 28-33. Grondahl \& Son, Oslo (1881) (1824, in French)

69. Stević, S.: Solvable subclasses of a class of nonlinear second-order difference equations. Adv. Nonlinear Anal. 5(2), 147-165 (2016)

70. Stević, S.: Sixteen practically solvable systems of difference equations (to appear) 